\title{
A Combined Simulation Approach to Evaluate Overtaking Behaviour on Two-Lane Two-Way Rural Roads
}

\author{
Valentina Branzi $\left(\mathbb{D},{ }^{1}\right.$ Monica Meocci $\mathbb{D}^{1},{ }^{1}$ Lorenzo Domenichini $\mathbb{D}^{1}{ }^{1}$ \\ and Margherita Calcinai ${ }^{2}$ \\ ${ }^{1}$ Civil and Environmental Engineering Department, University of Florence, Via S. Marta 3, 50139 Firenze, Italy \\ ${ }^{2}$ Tecne SPA-Gruppo Autostrade per l'Italia, Via dei Valtorta, 48, 2017 Milano, Italy \\ Correspondence should be addressed to Monica Meocci; monica.meocci@unifi.it
}

Received 17 March 2021; Accepted 12 August 2021; Published 21 August 2021

Academic Editor: Mohamed Hussein

Copyright (c) 2021 Valentina Branzi et al. This is an open access article distributed under the Creative Commons Attribution License, which permits unrestricted use, distribution, and reproduction in any medium, provided the original work is properly cited.

\begin{abstract}
A significant percentage of road fatalities and injuries occur in the nonmotorway rural road network. One of the main causes of accidents on these roads is represented by overtaking, as, by its nature, it involves a risk of a head-on collision with oncoming traffic. The paper describes a combined simulation approach (driving simulator and traffic microsimulation) designed to examine the influence of different traffic conditions on passing manoeuvres on two-lane two-way rural roads. The main focus was the evaluation of the end of the passing manoeuvre because it reflects the risk of a head-on collision. In addition, the study aimed to assess the usefulness of the proposed combined approach in the ability to proactively and quickly diagnose traffic safety problems and consequently to evaluate appropriate solutions. The data collected with an interactive driving simulator on a sample of 54 participants have been used to adjust some input data of the traffic microsimulation software. A specific situation consisting of a stationary heavy vehicle obstructing the entire lane was repeated in both experiments. The analyses focused on time-to-collision (TTC), defined as the remaining gap between the passing vehicle and the oncoming vehicle at the end of the passing manoeuvre. The results showed that the type of manoeuvre performed is significantly influenced by the traffic condition. Furthermore, the manoeuvre is influenced by the gap between two successive vehicles in the opposite lanes. Focusing on the end of the manoeuvre, it was found how a traffic increase leads to a significant reduction of the TTC values. Furthermore, the comparative analysis conducted between the data recorded following the combined approach and those obtained using exclusively the input data of the microsimulation software supports the usefulness of the proposed methodology for conducting road safety analyses, especially in complex traffic environments where drivers' behaviour plays a decisive role.
\end{abstract}

\section{Introduction}

Most road accidents worldwide occur on rural roads. They are associated with the highest risk of fatalities and serious injuries in the 41 OECD (Organization for Economic Cooperation and Development) countries [1] and accident data from other countries as the United States of America (USA) [2] and Australia [3] show comparable figures. Focusing on the European road network, between 2006 and 2015 , about 180,000 people were killed in accidents on roads outside urban areas, excluding motorways. This number represents $55 \%$ of all road fatalities in the EU [4]. In Italy, the situation is slightly less serious. In 2019, road accidents reported on rural roads amount to 36,107 (21\% of the total) with 1,532 people killed (48.3\%) and people injured $57.581(23.9 \%)$ [5].

Two-lane, two-way rural roads (i.e., road consisting of two opposing lanes of undivided traffic, in which lane changing and passing is possible only in oncoming traffic) constitute worldwide an important part of the rural road network. On these types of roads, overtaking plays an important role in influencing both road safety and mobility, in terms of capacity and level of service. While the lack of the ability to overtake may lead to the formation of large queues [6], the interaction with oncoming traffic flow from the opposite direction may significantly increase the risk of accidents, for example, due to the false temporal and distance estimations regarding the oncoming vehicles made by 
drivers [7-10]. Overtaking represents one of the most serious causes of two-lane two-way rural roads injuries and fatalities; due to its nature, it involves a risk of a head-on collision with oncoming traffic, and therefore it has a direct impact on safety. Previous studies showed that the accident severity resulting from this type of manoeuvre is significantly higher than that of other accident types on two-lane two-way rural roads [11-13].

In general, overtaking manoeuvre is one of the most complex and difficult driving processes which requires complicate decisions, involves a series of sequential actions (such as lane-change manoeuvres, possible acceleration and deceleration actions, and estimation on the relative speed of the overtaking and overtaken vehicles) and can fail in several ways. All these issues are undoubtedly amplified on two-lane two-way roads, mainly due to the variety of influencing factors. The drivers who overtake have to go through certain decision-making processes to accept or reject gaps presented to them in the oncoming traffic stream and to determine whether passing is justified under the constantly changing road and traffic conditions [14, 15]. The overtaking manoeuvre, indeed, can be performed in different ways, mainly based on the speed of the vehicle to be overtaken and the oncoming traffic in the opposite direction.

The overtaking manoeuvre was examined focusing on two aspects: (a) the development of models capable of explaining the different factors that can influence this manoeuvre and (b) the analysis of driving behaviour during the overtaking conducted in different conditions. In both cases, the analysis data were collected through in-field and naturalistic driving studies and, recently, using simulation techniques, especially by means of driving simulators.

The first relevant data to develop the overtaking manoeuvres models were obtained by observation and field study by recording video from external fixed positions of passing zones using only one camera in each passing zone $[16,17]$ and, more recently, using six cameras installed at a fixed point, next to passing sections $[18,19]$. In both cases, although data were recorded according to traffic volumes ranging between 300 and 1,000 vehicles per hour, results were not provided for different traffic volumes, as only in a few manoeuvres did an opposing vehicle occur approaching the vehicles passing and getting in the way.

Other researchers $[20,21]$ carried out naturalistic driving studies using instrumented vehicles to evaluate the driver's behaviour and performance (in terms of assessing collision risk with either the oncoming car) in real-time, by analysing surrogate safety measures.

Field and naturalistic data undoubtedly provide a rich source of data related to real-world experience; however, they require significant costs, involve safety problems for drivers and observers and difficulties in controlling experimental conditions, and may not provide satisfactory information for a full experimental design.

The interdisciplinary approach based on driving simulations represents a promising alternative method, which offers advantages in terms of cost reduction, ease of data collection, and completely safe and controlled experimental conditions. In addition, several studies have shown the reliability of the acquired data (i.e., the correspondence between the driver behaviour in the simulator and in the real world) in different driving environments, traffic situations, etc. [22-27], enough to make this approach a valid and reliable alternative to study complex manoeuvres.

In this context, some studies have used driving simulators to analyse and identify factors that influence driving behaviour during this critical manoeuvre, such as speed [11], the influence of different traffic-flow conditions [28], the visual-motor control strategies, and cognitive decisionmaking of drivers [7, 29]. Other researchers, instead, used driving simulator data to develop models to estimate the passing sight distance [30], the passing gap acceptance [31], the passing duration and passing distance [32], and the probability of head-on collisions that result from unsuccessful passing manoeuvres [33].

Although the approach adopted in these studies seems to be very promising, there are some disadvantages, mainly concerning the number of participants for each trial. In fact, in general, the driving simulator studies are carried out on samples composed of a limited number of subjects and consequently, the collected and analysed data are also limited and may not provide a complete generalization of the phenomenon studied.

An innovative method could be to use a combined approach based on the driving simulator and the microscopic traffic simulator. Indeed, the integrated simulation approach can provide a more realistic simulation platform for modelling conflicts [34].

Microscopic traffic simulators are powerful tools that, through the implementation of behavioural models, allow reproducing and predicting the evolution of different traffic situations and obtaining a multitude of data by generating various simulations.

Traditionally, traffic simulation models have been used to evaluate traffic system performance, and just more recently, there has been a growing interest in developing traffic simulation models to study the impact of traffic on road safety. Initially, the simulation-based approach offers the ability to proactively and quickly diagnose traffic safety problems and evaluate appropriate initiatives [34]. The microscopic model allows the estimation of road safety performance through a series of indicators, which represent real-time interactions between different pairs of vehicles belonging to the traffic flow. This approach is mainly based on the Traffic Conflict Technique (TCT) [35]. The TCT consists of capturing the near-misses conflicts and assessing the frequency and severity of collisions objectively and qualitatively, through the use of the so-called surrogate safety measures [36]. The use of these measures, which are not based on the observation of actual crashes, but instead on the probability of crashes, is proving increasingly interesting and promising for modelling and estimating safety, and it has a clear advantage over using crash data. Indeed, the use of crash data is a reactive approach that takes longer as data is infrequent, as well as being often of poor quality and underestimated. According to Gettman et al. [37], the ratio between conflicts and actual crash frequencies is generally in the range of thousands to 1 (depending on the definition of conflict). 
Some studies have integrated these two simulation tools (driving simulator and microscopic traffic simulator). However, the purpose of this integration was oriented to develop and implement a real-time running traffic simulation model capable of generating and simulating surrounding vehicles in a driving simulator. Focusing on the analysis of overtaking manoeuvres, only Jenkins and Rilett [38] developed a prototype that combined the microscopic traffic simulation program VISSIM with the driving simulator. The methodology of integrating simulations was applied to study the impact of both the length and the speed of the impeding vehicle on passing behaviour. By comparing the results from the passing experiments with field observations, the authors demonstrated some benefits of this approach, including the ability to generate specific vehicle volumes in the driving simulator and the ability to acquire comprehensive data.

Although the potential benefits of this approach have been recognized, the incorporation of the performance safety measures into traffic simulation models has been slow. Consequently, road safety simulation models are still being investigated [39]. In particular, there is still a significant obstacle in the development and application of the simulation model to assess traffic safety on rural roads [34]. Most studies concern intersections and most applications focus on modelling rear-end conflicts using car-following theory, but no studies appear to consider head-on collision on two-lane two-way roads [34].

All currently available microsimulation software packages are based on different submodels that explain how the drivers make decisions, in terms of route choice, car following, and lane selection, which are repeatable across the entire road network. All drivers' behaviour models consist of parameters, allowing users to enter input values within a specified range. These values may vary significantly based on driving conditions and geographical location (i.e., the local traffic characteristics and traffic conditions for a specific area). Thus, the default values for such variables must be adjusted for a realistic replication of local driving conditions [40].

The data recorded in virtual reality, which are representative of driving behaviour in specific, controlled, and safe driving conditions, could therefore be used for this purpose, that is, to "adjust" some of the default parameters characterizing the models of which the microscopic traffic simulators are composed. In this way, the advantages of both simulation tools could be fully used: the possibility of carrying out road safety analyses of complex situations on a considerable amount of data, using traffic microsimulator models whose parameters are representative of the drivers' behaviour in the geographic area and driving conditions analysed. However, this approach appears to be unexplored. This research opportunity motivates our study.

\section{Research Objective and Motivation}

The purpose of this study is to evaluate the influence of different traffic-flow conditions on the occurrence of potential risk situations, which could lead to a crash event, during the passing manoeuvre on two-lane two-way rural roads, using a combined simulation approach. Specifically, this paper focused on the analysis of the end of the passing manoeuvre, since up till this moment, there is still a risk of collision, therefore, reflecting the risk of head-on collisions (i.e., a collision with the opposite vehicle) and also because not many studies focused on the detailed analysis of the link between passing manoeuvres and head-on collisions in relation to traffic flows in the opposite direction.

Several studies analysed the passing manoeuvres on this type of road, mainly based on field and driving simulation studies. All these studies showed that one of the most critical factors involved in passing behaviour is the drivers' ability to estimate the required gap for passing a lead vehicle in front of an oncoming one. Specifically, they revealed that drivers' accepted passing gaps are widely influenced by several factors (such as the type of overtaking manoeuvre that a driver intends to perform, the traffic conditions in the opposite direction, and/or the waiting time for an opportunity for overtaking) which clearly suggest that drivers adapt their accepted gaps to overtake, depending on the traffic situation [21]. However, in literature, there are a few contributions $[28,32,41]$ that provide indications of the influence of different traffic volumes on the driver's behaviour during the overtaking manoeuvre. Overall, all three studies highlighted, as is conceivable, that the amount of oncoming traffic significantly affects the driving behaviour and the kind of manoeuvre adopted by the driver. While the studies conducted by $[29,32]$ were able to show this result only at the end of the passage manoeuvre (evaluating only this phase), the work in [28] extended this result, proving that traffic significantly influences the behaviour of drivers in all three phases of the passing manoeuvre analysed (beginning of the manoeuvre, occupation of the left lane, reentry in the right lane). Specifically, it revealed that the manoeuvres carried out are all the riskier, the greater the traffic volume.

Compared to these previous studies, a novel and peculiar feature of our study is to use both driving simulation and microscopic traffic simulations. In particular, the present study used the LaSIS driving simulator to better understand the interaction between the overtaking driver and the oncoming traffic, in order to obtain more reliable behavioural data. The results of driving simulator experiments are then used to adjust some driving behaviour parameters of the microscopic passing manoeuvre model for two-lane rural roads specially developed for the Aimsun microsimulation software [42], in order to effectively represent the driving behaviour during the overtaking manoeuvre on these types of roads.

Finally, in order to be able to provide the first suggestions on the potential for using such an approach, the traffic simulations conducted by the Aimsun software were also run with the software default values (without any adjustments). The results of this simulation and those obtained from the one in which some parameters were set according to the data recorded in the simulator were compared by statistical analysis to assess whether there were significant differences. 


\section{Aimsun Microscopic Passing Manoeuvre Model for Two-Lane Rural Roads}

The Aimsun (Advanced Interactive Microscopic Simulator for Urban and Non-Urban Networks, http://www.aimsun. com) microsimulation software [42] was used because of its ability to appropriately represent road network geometry and model in detail the behaviour of individual vehicles to reproduce explicitly traffic signal control plans, pretimed and actuated, and because it has an easy-to-use graphical user interface. Also, a particular feature of Aimsun is its ability to capture the empirical evidence that driver behaviour often depends on local circumstances (i.e., the acceptance of speed limits on-road sections, the influence of gradients, driver interaction whilst travelling in adjacent lanes).

Three main behavioural models are implemented on the base of the software operation, which allow describing the behaviour of the single vehicle reproducing the real traffic situations in the conditions of (a) car following, (b) lane changing, and (c) gap acceptance. In addition to these three main models, other models are implemented within the software for particular contexts and derivations from the main models themselves.

Recently, Llorca et al. [43] developed and calibrated a passing manoeuvre model in Aimsun [42]. In particular, it has been implemented in Aimsun 8.0.3. This model, used in the present work, covers the desire, decision, and execution processes of passing manoeuvres and relied on 14 parameters, defined specifically for the two-lane highways model. The 14 model parameters are associated with the experiment, the vehicle type, or the section Aimsun editors.

The parameters that affect the entire experiment are (1) delay time threshold for passing decision (delayTh) (s); (2) minimum speed difference threshold (mindV) $(\mathrm{km} / \mathrm{h})$; (3) maximum speed difference threshold $(\operatorname{maxdV})(\mathrm{km} / \mathrm{h}) ;(4)$ maximum rank in the platoon to desire to pass (maxRank) (vehicles); (5) number of simultaneous passes allowed (maxSimul) (passes); (6) delay between simultaneous passes (delaySimul) (s); (7) sensitivity factor for reduced car-following (RCF); (8) passing vehicle speed enhancement (PVSE); (9) speed difference threshold for enhanced passing vehicle speed (PVSETh) $(\mathrm{km} / \mathrm{h})$; and (10) remaining time to the end of the highway segment threshold (remainingtimeTh) (s). Traffic demand in Aimsun may be divided into several vehicle types.

The parameter that affects a particular vehicle type is safety margin for passing manoeuvre (safetymargin), defined by its minimum, maximum, mean, and standard deviation values (values in s).

The parameters that affect a single section are (1) mirror section identification (MirrorID); (2) available sight distance at the end of the passing zone (ASD) (m); and (3) sight distance factor (SDfactor).

\section{Methodology}

4.1. Overall Framework. This study proposed an integrated methodology that combines virtual reality driving simulation with traffic microsimulation. Figure 1 illustrates the

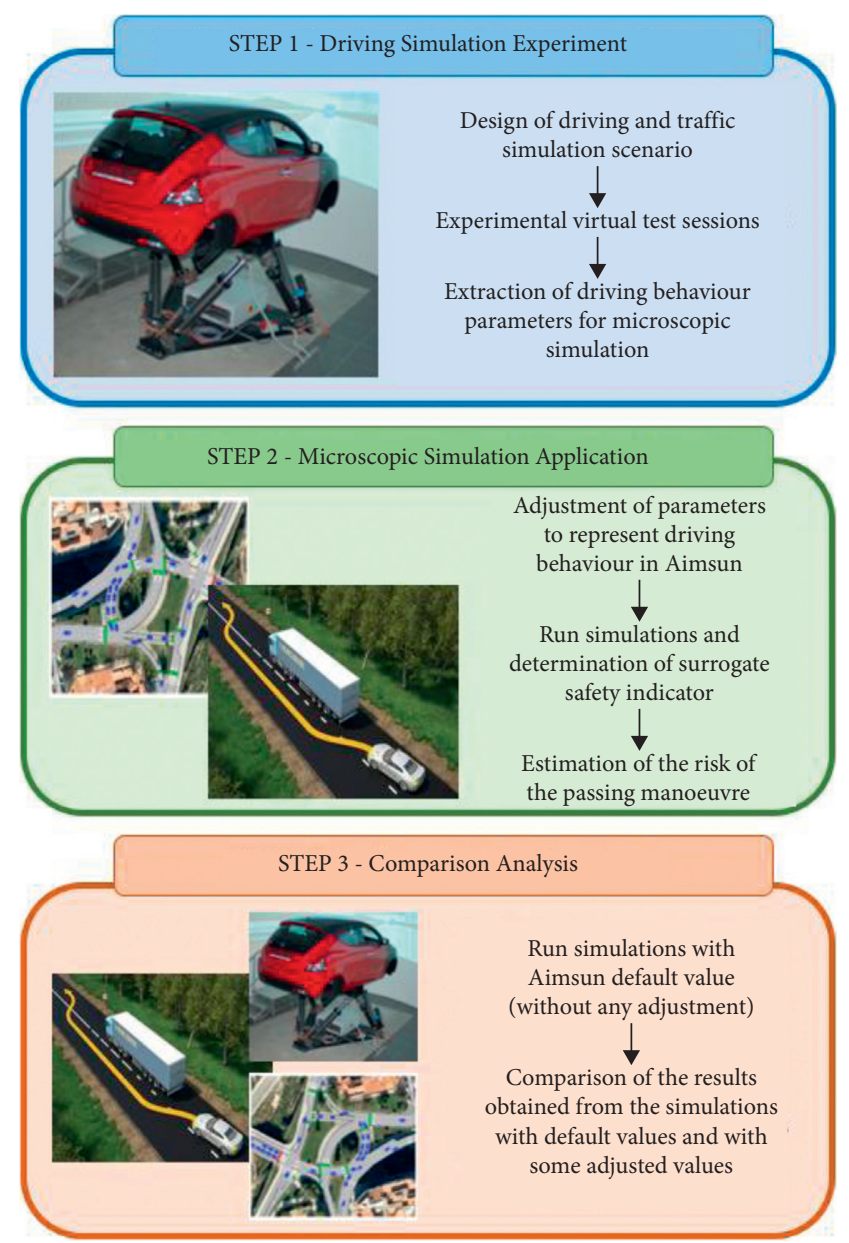

FIGURE 1: The overall framework of the proposed methodological approach.

overall design of the methodological approach proposed. It consists of three steps: (1) driving simulation experiments, (2) microscopic traffic simulation, and (3) comparison analysis. A driving simulation experiment is carried out to obtain information regarding driving behaviour during the overtaking manoeuvres under different traffic-flow conditions. The driving simulation environment allows capturing various driving behaviour patterns in a scientific and systematic manner. Therefore, the results help to gather different driving performances and behaviours that characterize different subjects belonging to a population. The results of this first step were further processed to adjust some parameters of microscopic traffic simulations. In the second step, Aimsun was used to assess the risk, in terms of safety, of the passing manoeuvres in different traffic-flow conditions. Some driving behaviour parameters of Aimsun were modified based on the results of the driving simulation experiment in step 1 . In the traffic simulation, three different traffic-flows conditions were also set and the consequent change in vehicle manoeuvres was obtained and recorded. The change in driving performances due to traffic-flow conditions was assessed taking into account a surrogate safety measure. Finally, in the last stage (step 3), further microscopic traffic simulations were carried out with the 
default values. The results obtained from these last simulations were processed and analysed according to phase two, to compare them with those obtained from the simulations in which some driving behavioural parameters were adjusted and to evaluate any statistically significant differences.

\subsection{Driving Simulation Experiment}

4.2.1. Apparatus. The LaSIS driving simulator used in this study is a motion-base simulator, equipped with a full-scale vehicle fitted on a $6^{\circ}$ of freedom Stewart's platform, allowing roll, yaw, and pitch. The vehicle interior is identical to the commercial version and it includes all commands normally available in such kinds of cars, with the steering wheel with force feedback. The cabin is surrounded by a cylindrical screen about $200^{\circ}$ wide, on which 4 projectors reproduce the driving environment. Sounds and noise of traffic in the environment and of the participant's car are generated by a multichannel audio system. The data acquisition frequency of the apparatus is $20 \mathrm{~Hz}$. The apparatus was previously validated as a reliable tool to predict the driver's behaviour in the real world and used to evaluate the driver's performance in terms of speed, acceleration/deceleration, and lateral position under various road environments and driving conditions $[22,23,44-46]$.

4.2.2. Simulated Road Scenario. About $50 \mathrm{~km}$ of a two-way two-lane rural road, with a posted speed limit of $80 \mathrm{~km} / \mathrm{h}$, was designed and implemented in the LaSIS driving simulator. It should be noted that although, according to the Italian Highway Code [47], the speed limit for the type of simulated roads is $90 \mathrm{~km} / \mathrm{h}$, it was cautiously chosen to set it equal to $80 \mathrm{~km} / \mathrm{h}$ based on the analysis of the data collected on the main two-way two-lane rural road located in the province of Florence, where the maximum limit recorded was $70 \mathrm{~km} / \mathrm{h}$.

The cross section of the carriageway is composed of two lanes (one for each direction), each $3.75 \mathrm{~m}$ wide and $1.50 \mathrm{~m}$ wide shoulders according to [48].

The road section was designed without any longitudinal grade and with few curves and no intersections, in order to exclude any possible influences of the sight distance on the driver's behaviour during passing manoeuvres. The driving experiments were carried out during daylight conditions and with good weather conditions (dry pavement). The passing was permitted only in the straight sections. The sections where the passage was not allowed were marked.

During the simulated route, the participants drove through four different analysis configurations, depending on the gap in the traffic in the opposite direction. Each analysis configuration was preceded by approximately $7 \mathrm{~km}$ of the standard route, where the road layout and traffic did not require the driver to change the desired driving style. Four different scenarios were built. The sequence of the four analysis configurations was counterbalanced to avoid influences due to the repetition of the same order in the experimental conditions.
The virtual scenarios were characterized by autonomous traffic, made with different vehicles, organized as "swarm" around the interactive vehicle. For each configuration, in the preliminary section of virtual road $(7 \mathrm{~km}$ of the standard route) the autonomous traffic was organized in both directions, while, in the examined section, it was only in the opposite direction to the subjective vehicle to avoid affecting the drivers' performance, in terms of speed, acceleration, deceleration, and so forth.

The situation that participants encountered in each of the four analysis configurations is shown in Figure 2. The subject vehicle was passing an impeding vehicle (front vehicle) while other vehicles were approaching from the opposite direction. The front vehicle was represented by a stopped heavy vehicle that simulated the situation of a vehicle in mechanical failure forced to obstruct the entire traffic lane due to the absence of a lay-by where you take refuge and wait for help. It was decided to obstruct the entire traffic lane (despite the possibility that the heavy vehicle occupied the shoulder) in order to reproduce the same situation that could be simulated in the Aimsun software. In fact, the Aimsun simulation does not allow the evaluation of the lateral movement of the vehicle, but it only identifies whether the vehicle is positioned in one or the other traffic lane, and it considers the obstruction of the entire lane.

The traffic approaching from the opposite direction consisted of the same platoon made up of six light vehicles; thus, the conditions to which the participants were subjected were the same in each configuration and there were no further variables (albeit of a minor entity, such as the type of vehicles and the colour of the vehicles) that, in some way, could affect the driver's performance and behaviour.

The passing gaps, which are the gap between the first and second vehicles of the platoon, were defined by the time spacing between these two vehicles at the time the subject encounters the lead opposite vehicle as illustrated in Figure 2. These passing gaps took on a different value in each analysis configuration, while the gap between the other vehicles of the platoon was set equal to 2 seconds in each configuration examined. The aim was to induce the driver to overtake by exploiting the first available gap; in fact, if the driver was unable to exploit this gap, he/she was forced to stop behind the stopped vehicle and wait for the whole platoon to pass, as a gap of 2 seconds did not allow overtaking.

An increase in passing gap was simulated for the four analysis configurations: a "critical" situation, a "precautionary" situation, and two other intermediate situations. The "critical" situation represented the space needed to physically perform the overtaking manoeuvre [49], while the "precautionary" situation represented the space needed to perform the overtaking manoeuvre in safety, according to Italian regulation on road design [48]. The "critical" and "precautionary" passing gaps were equal to 8 seconds and 20 seconds, respectively. They have been determined assuming that the driver maintains a speed corresponding to the imposed speed limit (equal to $80 \mathrm{~km} / \mathrm{h}$ ). Two passing gaps of 12 seconds and 16 seconds were chosen as intermediate situations. Table 1 summarizes the four different traffic conditions that the user has faced along the virtual road. 


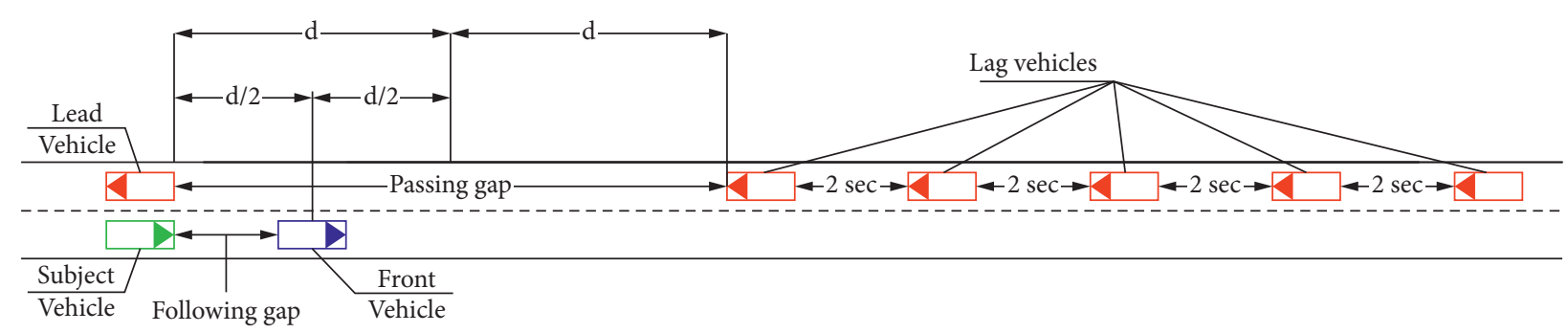

Figure 2: Passing gap configuration.

TABLE 1: Characteristics of the analysis configurations.

\begin{tabular}{lccc}
\hline Configuration & Traffic condition & $\begin{array}{c}\text { Passing gap } \\
(\mathrm{s})\end{array}$ & Passing gap (m) \\
\hline 1 & Critical & 8 & 178 \\
2 & Intermediate low & 12 & 267 \\
3 & Intermediate high & 16 & 356 \\
4 & Precautionary & 20 & 444 \\
\hline
\end{tabular}

It is important to note that, except for the subject vehicle, the speeds of all vehicles and the route they followed in the scenarios were previously programmed to be constant to allow the correct size and distribution of the passing gaps analysed.

4.2.3. Participants and Test Protocol. The minimum sample size was determined according to Cohen's theory [50], given the desired probability level, the anticipated effect size, and the desired statistical power level. The anticipated effect size $\chi^{2}$ was assumed equal to 0.6 ; the statistical power level and the $P$ value were set, respectively, equal to $90 \%$ and $5 \%$ (according to the previous studies, $[45,51])$. Using these parameters, the minimum sample size is sixty-five. Based on these results, sixty-five subjects (29 women and 36 men) were recruited voluntarily among students, staff of the University of Florence (Italy), and other volunteers from outside the University according to the following criteria: possession of a valid Italian driver's license, with at least five years of driving experience, an annual driven distance greater than $5000 \mathrm{~km}$, and low susceptibility to motion sickness. In order to avoid any bias of the outcomes, eventually related to the inexperience (young drivers) and aging (elderly drivers), the subjects aged less than 25 years and the subjects aged more than 65 years were excluded from the experimental study.

Five selected participants ( 2 women and 3 men) subsequently withdrew from the study and six subjects (5 women and 1 man) exhibited simulator sickness and did not complete the experiment. Thus, fifty-four subjects (22 women and 32 men) participated in the research, whose main characteristics are indicated in Table 2.

The selected participants were all residents of Tuscany and they had a normal or corrected-to-normal vision.

A chi-square goodness-of-fit test was conducted to determine whether the participants recruited were representative of the driver population resident of Tuscany.
Specifically, it was analysed whether these had the same proportion, in terms of age (according to the 4 age groups indicated in Table 2) and sex. The test was performed at a level of significance of $5 \%$. The results indicated that age and gender were similarly distributed among the participants recruited to the study as the Tuscan driver population $\left(\chi^{2}(7)=9.348, P=0.229\right)$.

All participants followed the same test protocol, explained below. The driving simulation experiment consisted, after signing the informed consent form, of the following steps: (a) communicating to the participants some basic information on the use of the simulator, as well as advising them to drive and to behave like in real-life situations, warning about possible simulator sickness and saying that they could stop the test at any time; (b) training phase to familiarize with the interactive vehicle and its control instruments; (c) a rest of $5 \mathrm{~min}$ to restore psychophysical conditions similar to those at the beginning of the test; (d) experimental phase in which each participant drove the simulation scenario with a specific sequence of traffic condition configurations.

Each participant encountered each of the four traffic condition configurations in random order to reduce the biases within the data collection. The participants were tested individually and were not briefed about the objectives of the research. Their participation was voluntary and no monetary reward was given for their involvement, which lasted about $45 \mathrm{~min}$.

\subsubsection{Extraction and Processing of Driving Behaviour Data.} Two of the 14 parameters that characterized the Aimsun passing manoeuvre were selected in the present study as those most influenced by the driver's behaviour when overtaking a stopped vehicle: "PSVE" and "safetymargin" parameters. The first is defined as the ratio between the maximum speed maintained by $5 \%$ of drivers at the end of the passing manoeuvre and the posted speed limit. It takes into account the possibility of travelling at a speed higher than the driver's free-flow desired speed. The second is defined as the safety margin for the passing manoeuvre and it is the parameter by which the risk of the manoeuvre is assessed. It represents the time to collision (TTC) between the passing vehicle and the opposite vehicle at the end of the overtaking process (i.e., the remaining gap between the two vehicles considered). When it reaches a value below a certain threshold (precisely considered "safety margin"), overtaking can be risky. Specifically, if the sum of the value of this 
TABLE 2: Participant characterization according to the factors considered.

\begin{tabular}{|c|c|c|c|c|c|c|}
\hline & & $25-34$ years & $35-44$ years & $45-54$ years & $55-64$ years & Total \\
\hline Sample consistency & & 14 & 17 & 15 & 8 & 54 \\
\hline \multirow{2}{*}{ Gender } & $\mathrm{F}$ & 7 & 6 & 5 & 4 & 22 \\
\hline & M & 7 & 11 & 10 & 4 & 32 \\
\hline \multirow{2}{*}{ Age } & MV & 27.6 & 39.6 & 48.9 & 57.4 & 38.5 \\
\hline & $\mathrm{SD}$ & 2.5 & 2.8 & 2.8 & 1.5 & 9.7 \\
\hline \multirow{2}{*}{ Driving experience (years) } & MV & 9.6 & 21.6 & 30.9 & 39.4 & 20.5 \\
\hline & DS & 2.5 & 2.8 & 2.8 & 1.5 & 9.7 \\
\hline
\end{tabular}

$\mathrm{F}=$ female; $\mathrm{M}=$ male; $\mathrm{MV}=$ mean value; $\mathrm{SD}=$ standard deviation .

parameter and the time required to complete the manoeuvre is less than the TTC assessed at the start of the manoeuvre, the overtaking is considered not to be at risk and can be completed safely. Otherwise, the opposite is true. The "safetymargin" parameter, during microscopic traffic simulations, takes on a different value for each vehicle.

To evaluate the first parameter considered, the PVSE, defined by a scalar factor, the speed reached by each driver at the end of the passing manoeuvre was extracted from the raw driving simulator data. For the determination of the "safety margin," the TTC between the subject vehicle and the lead opposite vehicle was calculated at the end of each passing manoeuvre. The TTC is measured at the end of the passing manoeuvre (as there is still a risk of the collision up to this moment) and reflects the risk to collide with the opposite vehicle. The end of a passing manoeuvre was defined, for each driver, as the instant when the subject vehicle wholly turns back into his lane (in front of the impeding vehicle) corresponding to the instant in which the subject vehicle's rear left wheel touches the centre line (Figure 3).

It is important to point out that these measures were available only for "accepted" passing gaps, that is the situation in which the driver has believed the first available gap (i.e., the one between the first and the second vehicle coming from the opposite direction, as shown in Figure 2) was sufficiently wide and he/she has completed the passing manoeuvres. In fact, due to how the analysis configurations in virtual reality were built, if the drivers did not exploit the first available gap, they were forced to wait for the complete passage of the entire platoon and overtake the stopped vehicle when there was no traffic in the opposite lane (this situation, in the rest of the text, will be indicated as the one in which the gap is rejected).

In addition, the speed held by each participant in freeflow driving conditions was also extracted from the raw driving simulator data. It is the speed recorded along a road section where there were no road geometrical characteristics and traffic conditions that forced the driver to change his driving style and to adopt a speed other than the desired one.

\subsection{Traffic Microscopic Simulation}

4.3.1. Network Model Coding and Run Simulation. The road network consists of a single straight segment of one kilometre in length, without intersections, with a single carriageway and two traffic lanes (one for each direction). Each traffic lane is 3.75 meters wide. The posted speed limit is $80 \mathrm{~km} / \mathrm{h}$, and it is consistent with the scenario used in the virtual reality experiment.

The traffic demand was assigned through the use of the origin/destination (O/D) matrix, characterized by the following input data: centroids of origin and destination, vehicle types in the network and their attributes, vehicle classes, and the number of movements from each origin centroid to each destination centroid. For the experiment, two centroids were defined at the ends of the segment, and a single vehicle class consisting of light vehicles was defined. As regards the determination of the number of movements, three different matrices were defined, each associated with a different traffic flow to reproduce three different levels of service (LOS). In this way, it was possible to reproduce different traffic-flow conditions in the opposite direction to the subject vehicle and, thus, assess how the LOSs may affect the execution of the passing manoeuvre. Specifically, three traffic-flow values were considered corresponding to service levels $A, B$, and $C$ for a road with a speed limit of $80 \mathrm{~km} / \mathrm{h}$, according to the Highway Capacity Manual [52].

Two different traffic distributions were selected for the two different directions of travel. In the traffic lane where the stationary heavy vehicle was located, a uniform distribution was selected with a number of arrivals such that it did not create the phenomenon of multiple queuing behind the stationary heavy vehicle, while in the opposite direction, an exponential distribution of arrivals was selected.

As regards the input data relating to vehicle classes, in addition to some behavioural parameters recorded through the driving simulator, it was decided to adjust other two input data of the microsimulation model in order to obtain results closer to reality: (a) the reaction time and (b) the maximum acceleration. Specifically, the value of 1 second has been assigned to the first parameter, according to Basak et al. [53] (the default value assumed by the software is 1.2 seconds), while, according to the acceleration/deceleration model defined by Bokare et al. [54], an average value of $1.2 \mathrm{~m} / \mathrm{s}^{2}$ and a standard deviation of $0.3 \mathrm{~m} / \mathrm{s}^{2}$ have been assigned for the second parameter (the default values are, respectively, $3 \mathrm{~m} / \mathrm{s}^{2}$ and $0.2 \mathrm{~m} / \mathrm{s}^{2}$ ).

Once the scenario described above was set up, it was decided to run the experiment using the Monte Carlo algorithm. Three different experiments were performed, each characterized by a different traffic demand, corresponding to the three LOS examined. 


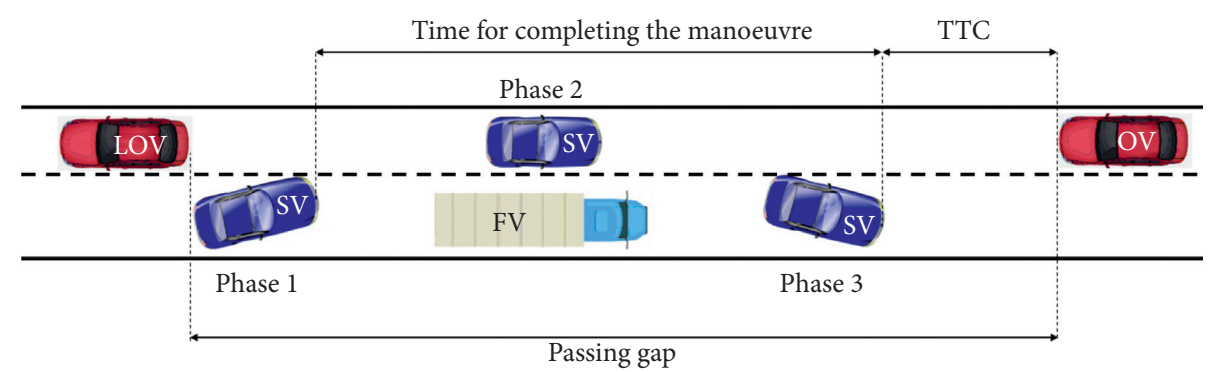

FIGURE 3: Passing manoeuvre phases (LOV= leading opposite vehicle; OV= opposite vehicle; $\mathrm{SV}=$ subject vehicle; $\mathrm{FV}=$ front vehicle).

A series of replicas were defined for each experiment to obtain a consistent data sample for subsequent analyses. Specifically, for each of the three experiments, the following replicas were carried out in order to obtain a comparable number of overtaking manoeuvres for the three different traffic conditions considered: (a) 12 replications for LOS A condition, (b) 36 replications for LOS B condition, and (c) 48 replications for LOS C condition. The different number of replicas for the different LOS conditions analysed is explained by the fact that it was necessary to generate a smaller number of vehicles in the lane where the stationary vehicle was in order not to create queuing phenomenon behind it.

4.3.2. Estimation of the Risk of Passing Manoeuvre. In order to assess the influence of different traffic-flow conditions on the risk of passing manoeuvre, the TTC was selected as a surrogate safety indicator. It is considered the most efficient and it is usually quantified using microscopic traffic simulations [55-57]. TTC is defined as the time remaining to the collision between two vehicles if they continue at their current speed and remain on their paths until the moment of the crash. Therefore, TTC is inversely related to accident risk: the smaller the value assumed by the TTC, the greater the probability that the conflict evolves into an accident (i.e., a null value of the TTC reflects a collision).

In this study, according to several similar types of research $[8,28,33,41,58-60]$, the minimum TTC to the opposite vehicle at the end of the passing manoeuvre was used as a head-on collision proximity measure. Specifically, the minimum TTC was defined as the remaining gap (in seconds) between the passing vehicle and the opposite vehicle at the end of the overtaking process. Mathematically, the TTC was calculated by the division of the distance between the fronts of the subject vehicle and the opposite vehicle by the sum of their speeds. The minimum TTC was the TTC value at the end of a successful passing manoeuvre.

Several threshold values have been suggested for TTC in the literature. In the present study, according to the information provided by [52], passing with a final clearance time of less than $2 \mathrm{~s}$ was deemed risky.

The data sample recorded during the traffic microsimulations was analysed (as previously described) by dividing it into six subsets, according to the traffic condition (LOS A, LOS B, and LOS C) and the type of manoeuvre performed. Preliminarily, by analysing the driving behaviour in the initial phase of the passing manoeuvres recorded during the traffic microsimulation (in particular by examining the speed), it was possible to distinguish them into two types: (a) flying passing manoeuvre (i.e., a pass in which the driver is not forced to slow down before making the pass, referred to as type "1" passing manoeuvre) and (b) passing manoeuvre after waiting for queues (i.e., when the user decides to stop behind the impeding front vehicle and wait for the first available gap to be able to pass it safely, referred to as type "2" passing manoeuvre).

Each subset was analysed in terms of both the gap accepted by the user to carry out the overtaking manoeuvre and TTC with respect to traffic coming in the opposite direction.

4.4. Comparative Analysis. To preliminary evaluate the effectiveness of the proposed methodology in carrying out safety analysis, further microscopic traffic simulations were performed with only Aimsun default values. Specifically, the results, obtained from these latter microscopic traffic simulations, were processed and analysed in the same way adopted in the second step (Figure 1), in order to compare them with those obtained from the microsimulations, in which some driving behavioural parameters were adjusted to evaluate any statistically significant differences.

The most popular statistical approaches for group comparisons (such as parametric tests for unpaired samples: independent $t$-test and ANOVA test) are typically used to examine the level of confidence about the hypothesis that the data of two groups of different samples belong to the same population. Among them, in the comparative analysis of the two considered traffic microsimulation applications, bilateral independent-samples Student's $t$-tests ( $t$-test) were carried out to determine the statistical significance of the results achieved. Specifically, for both dependent variables (gap and TTC), six bilateral independent-samples $t$-tests were run: one for each LOS and type of manoeuvre considered ( 3 LOS $\times 2$ types of manoeuvre). Preliminarily, it was verified that the data recorded can be analysed using this type of test, verifying the assumptions that must be considered (normality distribution and homogeneity of variances). In the null hypothesis (H0), the indicators (gap and TTC values) in the two data sets were considered to belong to the same population. The threshold for statistical significance was set at 0.05 . 


\section{Results and Discussion}

\subsection{Driving Simulator Results}

5.1.1. General Statistics. The resulting data set included a total of 216 passing gap observations (54 participants $* 4$ configurations of passing gaps). In two cases, these passing manoeuvres ended in a collision; both crashes occurred in the "critical" configuration and at the end of the passing manoeuvre (during the reentry phase). The drivers collided with the stationary heavy vehicle to avoid a head-on collision with the oncoming vehicle in the opposite direction. These two observations were removed from the estimation data sets and, thus, 214 passing gap observations were examined. Of these passing gaps, 134 (62.6\%) were accepted, while the rest were rejected.

Table 3 presents summary statistics of passing manoeuvres relating to the number of accepted and rejected gaps in the 4 different analysis configurations. The analysis of the type of behaviour approaching the heavy stopped vehicle (Table 3) understandably showed that, as the available gap increases, the number of gaps accepted by drivers also increases. Specifically, the results showed that, in the "critical" gap situation (8s), most of the participants (52\%) did not perform any manoeuvre and they preferred waiting behind the stationary vehicle. On the contrary, in presence of larger gaps, the percentage of the motorists who performed an overtaking manoeuvre increased considerably reaching $78 \%$ in the configuration " 4 " (available gap equal to $20 \mathrm{~s}$ ).

The Chi-squared test results showed that there was a statistically significant association between configuration and the type of manoeuvre chosen by the driver (no passing/ passing) $\left(\chi^{2}(3)=11.928\right.$ and $\left.P=0.008\right)$. The association was moderately strong according to Cohen [50] (Cramer's $\mathrm{V}=0.235$ ). Therefore, the considered passing gaps significantly affected the driver's manoeuvre approaching the heavy stopped vehicle.

\subsubsection{Driving Behaviour Parameters for Microscopic Simulation}

(1) PSVE. To determine the PVSE parameter, the speed recorded at the end of the overtaking manoeuvre was extracted for each participant in each analysis configuration. The mean speed and the standard deviation of acquired data are summarized in Table 4.

The results revealed that the average value of all speeds recorded at the end of the overtaking manoeuvre (considering all configurations) was $71.58 \mathrm{~km} / \mathrm{h}(\mathrm{DS}=12.55 \mathrm{~km} / \mathrm{h})$. Specifically, it was found that the speed at the end of the analysed manoeuvre increases as the gap available in the opposite traffic flow increases. The speed increase recorded is about $22 \mathrm{~km} / \mathrm{h}$ (i.e., $38 \%$ ) between configurations " 1 " and " 4 ," whose speed at the end of the manoeuvre reached a value close to the posted speed limit. This is probably due to the way the drivers are overtaken based on the available gaps. In fact, it was found that, in the smallest gap configurations (8s and $12 \mathrm{~s}$ ), after passing the stationary vehicle, drivers were forced to turn sharply to reenter into their lane. This did not allow them to reaccelerate and to reach the desired speed. In the wider gap configurations (16 s and $20 \mathrm{~s}$ ); on the contrary, drivers overtook less abruptly, using these spaces to accelerate again, regain the desired speed, and get back into their lane.

It should also be noted that, as the available gap increased, higher standard deviations were recorded, highlighting a reduction in the homogeneous behaviour of the driver where the gap available to overtake increased.

In order to understand if these differences were attributable to the analysed configuration and were not random, a one-way repeated measures ANOVA test was conducted. There were no outliers and the data were normally distributed, as assessed by Shapiro-Wilk's test $(P>0.05)$. The assumption of sphericity was violated, as assessed by Mauchly's test of sphericity $\left(\chi^{2}(5)=15.683, P=0.008\right)$ and, therefore, a Greenhouse-Geisser correction was applied $(\varepsilon=0.720)$. The findings of the statistical test revealed that the configuration (i.e., the gap available to perform the passing manoeuvre) made statistically significant speed changes recorded at the end of the passing manoeuvre $(F(2.159,51.807)=48.457$, $P<0.001$, partial $\left.\eta^{2}=0.669\right)$. Post hoc analysis with a Bonferroni adjustment revealed that there are significant differences between all configurations $(P<0.001)$, except between the configuration " 3 " and the configuration " 4 " $(P=0.065)$.

Before determining the value of the PVSE parameter, the speed data observed at the end of the manoeuvre were organized into a histogram (Figure 4) and the Shapiro-Wilk's test was conducted to verify the goodness of fit of these data to the normal distribution. Specifically, it was assumed that the speed data are from a normally distributed population $(\mathrm{HO}=$ null hypothesis) if the resulting $P$ value was equal to or higher than 0.05 . The results showed that the speed data can be considered as fitting a normal distribution $(P=0.192)$.

Subsequently, the speed value adopted by $5 \%$ of users (95 ${ }^{\text {th }}$ percentile-V95) was calculated and, consequently, the value of the PVSE factor, as the ratio between the obtained value of V95 and the posted speed limit. The two parameters were, respectively, $92.23 \mathrm{~km} / \mathrm{h}$ (dashed red line in Figure 4) and 1.15 .

The PVSE value obtained is higher than the Aimsun default value set at 1.10 determined based on the overtaking data recorded during the field observations [43], thus showing that the speed recorded in the virtual reality experimentation at the end of the manoeuvre analysed is greater than that observed in the field. This difference may be caused by some differences in the situation analysed, in terms of the type of passing manoeuvre faced by the driver and the type of vehicle to overtake. While in the field study [43] the overtaking manoeuvre of a moving vehicle was examined, in the present virtual reality experimentation, the overtaking manoeuvre of a stationary heavy vehicle was instead analysed. This can presumably lead you to believe that it has changed the user's behaviour. In fact, while the situation of overtaking a vehicle in motion is a situation frequently faced by the driver in the real world, the situation analysed in this study represents an unusual condition that occurs much less frequently on Italian 
TABLE 3: Gaps accepted and rejected in the analysis configuration.

\begin{tabular}{lccc}
\hline Configuration & Passing gap (s) & No. of gaps accepted & No. of gaps rejected \\
\hline 1 & 8 & $25(48 \%)$ & $27(52 \%)$ \\
2 & 12 & $31(57 \%)$ & $23(43 \%)$ \\
3 & 16 & $36(67 \%)$ & $18(33 \%)$ \\
4 & 20 & $42(78 \%)$ & $12(22 \%)$ \\
\hline
\end{tabular}

TABle 4: Number of measurements, mean value, and standard deviation of speed in each analysis configuration.

\begin{tabular}{lccc}
\hline Configuration & No. of measurements & Mean value $(\mathrm{km} / \mathrm{h})$ & St. deviation $(\mathrm{km} / \mathrm{h})$ \\
\hline 1 (gap: $8 \mathrm{~s}$ ) & 25 & 57.88 & 7.92 \\
2 (gap: $12 \mathrm{~s}$ ) & 31 & 66.75 & 8.17 \\
3 (gap: $16 \mathrm{~s}$ ) & 36 & 75.47 & 9.66 \\
4 (gap: $20 \mathrm{~s}$ ) & 42 & 79.98 & 11.12 \\
All configurations & 134 & 71.58 & 12.55 \\
\hline
\end{tabular}

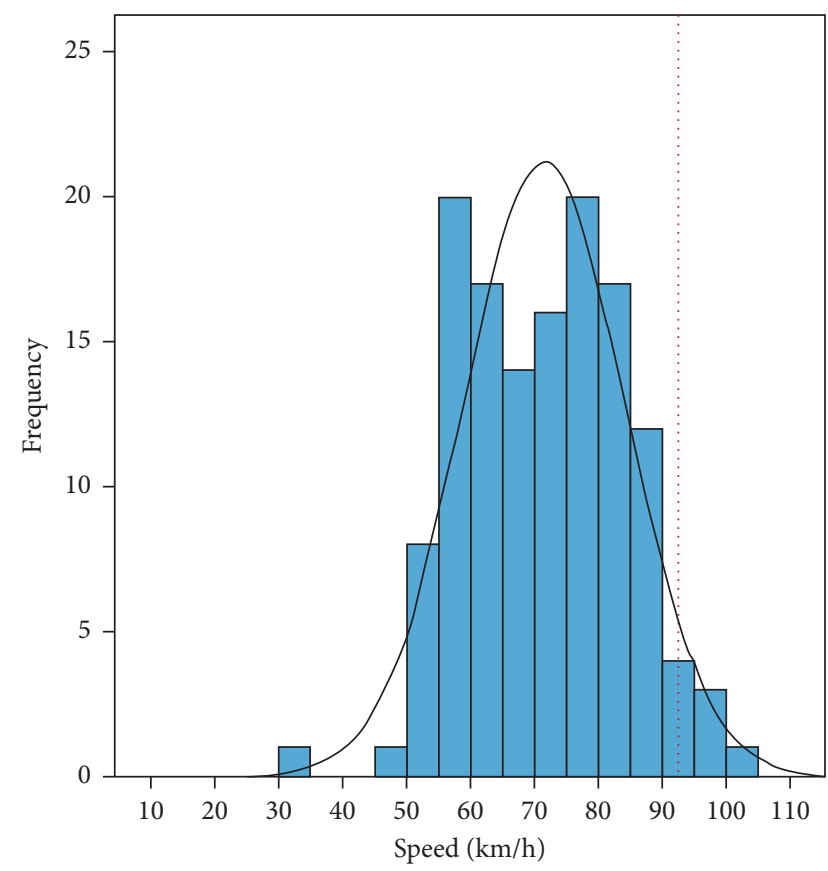

Figure 4: Histogram of speed data at the end of passing manoeuvre.

rural roads. Therefore, it is plausible that the driver, in this situation, has performed the manoeuvre in a less "quiet" and more sudden way due to the lack of habit in practising it. This hypothesis was confirmed by the results of the questionnaire filled out by the participants at the end of the virtual experiment; many of them declared that they had made a hasty decision on how to carry out the manoeuvre, as it was not clear to them whether the stopped vehicle was isolated or the last one in a queue.

Furthermore, since, in the experiment, the driver has to overtake a heavy vehicle (approximately twice the length of a light vehicle), he/she will presumably tend to assume a higher speed at the end of the manoeuvre, due to a longer acceleration phase held during the manoeuvre itself: the longer the vehicle to be overtaken, the greater the space in which the vehicle will have to accelerate.
(2) Safetymargin. As regards the evaluation of the "safetymargin" parameter, the value of TTC recorded for all the passing manoeuvres observed, for each analysis configuration, was determined. The descriptive statistics of observed data are summarized in Table 5.

The results highlighted a very different driver behaviour in the analysed configurations. A similar trend to that recorded for the speed values at the end of the passing manoeuvre can be observed. Specifically, it was found that the TTC value increases as the gap available in the opposite traffic flow increases. The maximum increase (corresponding to 2.47 seconds) was recorded between configurations " 1 " and " 4 ." It should also be noted that, as the available gap increased, higher standard deviations were recorded. It confirms the results described in the previous session, that is, a reduction of homogeneity in the driver behaviour where the gap available for overtaking has increased.

Only in low traffic conditions (configuration "4" characterized by the widest gap analysed), the driver turns back into the right lane with a mean value of TTC greater than 2 seconds, while in the other analysis situations the mean value of TTC is less than 2 seconds, resulting even below the second in the configurations characterized by the smallest available gaps (configurations "1" and "2"). Also, considering the four configurations, the average TTC at the end of the passing manoeuvre was less than 2 seconds $(1.56 \mathrm{~s})$. Furthermore, as can be seen from Table 5, approximately $69 \%$ of the observations (total observations 134) were less than $2 \mathrm{~s}$. In particular, in all the analysis configurations except for configuration " 4 ," a TTC value less than 2 seconds was recorded for most of the observed manoeuvres, even reaching $100 \%$ in configuration "1." Therefore, according to the AASHTO Manual [52] which recommends a minimum TTC value of $2 \mathrm{~s}$, as well as other studies that have classified risky passing manoeuvres with clearance gaps of less than $2 \mathrm{~s}$ $[61,62]$, the manoeuvres recorded in all the analysis configurations (except in the " 4 " configuration) are, on average, to be considered risky.

A one-way repeated measures ANOVA was conducted to determine if the differences in recorded TTC values, between the configurations, were statistically significant. 
TABle 5: Number of measurements, mean value, and standard deviation of TTC in each analysis configuration.

\begin{tabular}{lcccccc}
\hline Configuration & $N$ & $N_{\text {TTC }<2 s}$ & $\begin{array}{c}\text { MV } \\
(\mathrm{s})\end{array}$ & $\begin{array}{c}\text { SD } \\
(\mathrm{s})\end{array}$ & $\begin{array}{c}\text { Min } \\
(\mathrm{s})\end{array}$ & $\begin{array}{c}\text { Max } \\
(\mathrm{s})\end{array}$ \\
\hline 1 (gap $=8 \mathrm{~s})$ & 25 & $25(100 \%)$ & 0.39 & 0.20 & 0.02 & 0.78 \\
$2($ gap $=12 \mathrm{~s})$ & 31 & $29(94 \%)$ & 0.91 & 0.47 & 0.38 & 2.21 \\
$3($ gap $=16 \mathrm{~s})$ & 36 & $28(78 \%)$ & 1.43 & 0.60 & 0.40 & 2.75 \\
$4($ gap $=20 \mathrm{~s})$ & 42 & $11(26 \%)$ & 2.86 & 1.05 & 0.80 & 5.62 \\
$\begin{array}{l}\text { All } \\
\text { configurations }\end{array}$ & 134 & $93(69 \%)$ & 1.56 & 1.18 & 0.02 & 5.62 \\
\hline
\end{tabular}

$N=$ number of measurements; $\mathrm{N}_{\mathrm{TTC}}<2 \mathrm{~s}=$ number of measurements with TTC values less than 2 seconds; $M V=$ mean value; $S D=$ standard deviation.

There were no outliers and the data were normally distributed, as assessed by Shapiro-Wilk's test $(P>0.05)$. The assumption of sphericity was violated, as assessed by Mauchly's test of sphericity $\left(\chi^{2}(5)=38.000, P<0.001\right)$ and, therefore, a Greenhouse-Geisser correction was applied $(\varepsilon=0.556)$. The findings of the statistical test revealed that the configuration (i.e., the gap available to perform the passing manoeuvre) made statistically significant changes in TTC recorded $(F(1.669,40.048)=99.152, P<0.001$, partial $\left.\eta^{2}=0.805\right)$, showing that drivers adopted very different behaviours in terms of risk at the end of the manoeuvre, according to the changes in the traffic configuration. Post hoc analysis with a Bonferroni adjustment revealed that there are significant differences between all configurations $(P<0.001)$.

Few driving simulation studies are available in the literature to compare the obtained TTC values. Specifically, the comparison with these studies shows a very similar value with the TTC mean value obtained by [28], in which a value equal to $1.30 \mathrm{~s}$ was recorded, as well as a fairly appreciable difference (approximately 1 second higher than the value recorded in this study) with the study by Farah et al. [41]. This difference is probably due to the different configurations of the driving simulators since Farah et al. [41] used a low-cost driving simulator (steering and pedals not installed on a real vehicle, only one screen in front of the driver with a usual field of view of only $60^{\circ}$ ). Therefore, bearing in mind differences in equipment and traffic conditions used in the different experiments, our results are consistent with those obtained in previous studies carried out using driving simulators.

Based on the data obtained from the driving simulations (Table 5), the safety margin parameter for passing manoeuvre, defined by its average, standard deviation, minimum, and maximum values, is characterized, respectively, by the following values (in seconds): 1.56, 1.18, 0.02, and 5.62. These values differ from the Aimsun default values, which instead assume the following values, respectively: 5 , 5.8, 1, and 10. According to Llorca et al. [43], a minimum value of $1 \mathrm{~s}$ was chosen based on previous research $[17,61]$, while the maximum value was $10 \mathrm{~s}$, as longer safety margins were not considered reasonable by them even with very conservative drivers. The mean and standard deviation values were selected (by adjusting simulated and observed critical gaps with a probability of acceptance equal to 0.5 ) to be $5 \mathrm{~s}$ and $5.8 \mathrm{~s}$, respectively. These differences are attributable (according to the results of the questionnaire filled out by the recruited subjects), to the differences in the situation analysed in terms of the overtaking type to be performed (vehicle stopped and not in motion) and of the type of vehicle to overcome (heavy vehicle, rather than a light vehicle).

(3) Desired Speed. The desired speeds adopted by the drivers while driving in a road section where they were able to adopt the desired driving style were calculated, in order to characterize the drivers' behaviour while driving in "undisturbed" conditions.

Preliminary, the speed data observed were organized into a histogram (Figure 5).

The Shapiro-Wilk's test was conducted to analyse the appropriateness of the normal distribution to the desired speed data. The test results showed that the null hypothesis (data are from the hypothesized normal distribution), cannot be rejected at the 95\% confidence level $(P=0.864)$.

The means and standard deviation values of the free-flow speed are $92.70 \mathrm{~km} / \mathrm{h}$ and $9.38 \mathrm{~km} / \mathrm{h}$, respectively. This result is consistent with the indications of the Highway Capacity Manual [6], which provides an approximate estimate of the speed desired by the driver corresponding to the posted speed limit plus $10 \mathrm{mph}$ (approximately, $16 \mathrm{~km} / \mathrm{h}$ ) or corresponding to the design speed of the road. Due to the dependence of the desired speed to the posted speed limit equal to $100 \mathrm{~km} / \mathrm{h}$ [42], the average desired speed value obtained in the virtual reality simulation $(92.70 \mathrm{~km} / \mathrm{h}$ with standard deviation equal to $9.38 \mathrm{~km} / \mathrm{h}$ ) and that defined by the Aimsun default values (equal to $110 \mathrm{~km} / \mathrm{h}$ ) is not comparable. However, regardless of the posted speed limit, the standard deviation obtained by driving simulator results is consistent with the Aimsun default values, equal to $10 \mathrm{~km} /$ $\mathrm{h}$. Therefore, in this case, the findings show a similar distribution between the real behaviour and the lane-change model used in the Aimsun microsimulator [43].

\subsection{Traffic Microscopic Simulation Results}

5.2.1. General Statistics. The data set from the traffic microsimulations included a total of 1,114 passing gap observations. In $622(56 \%)$ on these gaps, the passing manoeuvre type "1" was performed (i.e., the stationary vehicle was passed, without waiting behind it), while in the rest 492 cases (44\%), a type "2" overtaking manoeuvre was observed. Table 6 shows summary statistics of observed passing manoeuvres based on the three LOS conditions considered. The results are consistent with those obtained from the experiments conducted in virtual reality. As traffic flow increases, the percentage of vehicles forced to queue behind the stopped vehicle and wait for the passing of the platoon coming in the opposite direction (before overtaking) increased; consequently, the percentage of flying pass manoeuvres observed decreased. Specifically, the results (Table 6) highlighted that, in LOS A traffic conditions, most of the vehicles $(74.3 \%)$ performed the passing manoeuvre, 


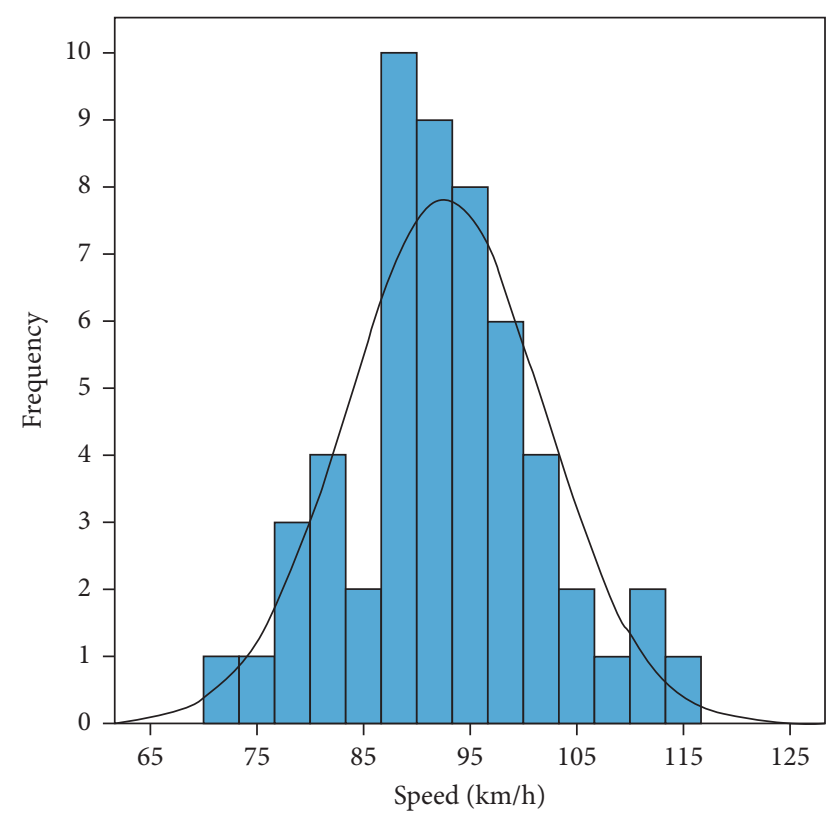

Figure 5: Histogram of free-flow speed data.

TABLE 6: Type of manoeuvres performed according to the different LOS analysed.

\begin{tabular}{lcccccc}
\hline & \multicolumn{3}{c}{ Type of passing manoeuvre } & \multicolumn{2}{c}{ Total passing } \\
LOS & \multicolumn{2}{c}{1} & & \multicolumn{2}{c}{2} & \multicolumn{2}{c}{ manoeuvre } \\
& $N$ & $\%$ & $N$ & $\%$ & $N$ & $\%$ \\
\hline A & 373 & 74.3 & 129 & 25.7 & 502 & 100 \\
B & 159 & 46.4 & 184 & 53.6 & 343 & 100 \\
C & 90 & 33.2 & 181 & 66.8 & 271 & 100 \\
\hline
\end{tabular}

$1=$ flying pass manoeuvre; 2 =overtaking manoeuvre, observed after waiting behind the stationary vehicle.

not performing any deceleration behaviour. This trend was completely reversed in the traffic condition represented by a LOS C (only $332 \%$ of the total passing manoeuvres observed were classified as flying pass manoeuvres), while an intermediate situation occurred in the LOS B traffic conditions. Although, in this situation, the percentage of overtaking carried out without stopping behind the stationary vehicle is still lower $(46.4 \%)$ to the one in which the opposite behaviour was observed (53.6\%).

The Chi-squared test results showed that there was a statistically significant association between traffic conditions and the type of passing manoeuvre performed (flying pass/ pass after stopping behind the stopped vehicle) $\left(\chi^{2}(2)=\right.$ 142.042 and $P<0.0001)$. The association was quite strong, according to Cohen [50] (Cramer's V=0.355). Therefore, the traffic-flow condition significantly affected the passing manoeuvre approaching the heavy stopped vehicle.

5.2.2. Risk Analysis of the Passing Manoeuvre. Data set recorded were preliminarily plotted into histograms in which the TTC frequency was correlated to the three different traffic-flow conditions, respectively, for the two types of passing manoeuvres analysed (Figure 6). It is possible to note that, in both manoeuvres, as traffic increases (i.e., as LOS decreases) the frequency of lower TTC values increases. In particular, for the most intense traffic conditions (LOS C), no TTC values higher than 10 seconds for type " 1 " passing manoeuvre and exceeding 12 seconds for type " 2 " passing manoeuvre were recorded. This is because high time gaps do not occur as traffic increases, as shown by the histograms in Figure 7 which represent the accepted gap distributions in the three different traffic conditions, respectively, for the two manoeuvres analysed.

Figure 7 highlights how, as traffic increases, the width of the most accepted gap decreases. Specifically, for LOS A, there is a fairly uniform distribution of the gaps included in the interval (4s-6s) for the type A manoeuvre and in the widest interval ( $8 s-14 s)$ for the type "2" manoeuvre. For LOS B and LOS C, on the other hand, the histograms show peaks shifted to the left, in the interval (4s-6s) for the type "1" manoeuvre and in the interval (6s-10 s) for the type " 2 ."

Table 7 summarizes the gap and TTC values (mean and standard deviation), as well as the results relating to manoeuvres considered at risk, that is, those for which a risk threshold TTC value has been recorded (set equal to $2 \mathrm{~s}$ following the Highway Safety Manual [52]). The results showed that, as traffic increases, the number of manoeuvres considered at risk increases. In fact, as the traffic flow increases, there are fewer gaps of such width as to guarantee the possibility of carrying out the manoeuvre safely, and the users who have to overtake, after having waited for a certain time for the gap useful to carry out the manoeuvre, are willing to accept gaps of a smaller size than is deemed appropriate. As a result, the TTC values are significantly reduced and the percentage of manoeuvres considered at risk increases. Specifically, between the situation of LOS A and that of LOS B, there was an increase in manoeuvres considered at risk of around $10 \%$ for both types of passing manoeuvres. While between the situation of LOS B and LOS $C$, there was a greater increase for type " 1 " passing manoeuvre $(8 \%)$ and a negligible increase in type " 2 " passing manoeuvre (2\%).

It was also found that, for passing manoeuvre " 1 ," greater manoeuvres at risk were recorded compared to those recorded for passing manoeuvre "2," in all traffic-flow conditions. This difference can be attributed to the different driver behaviour in the two different circumstances. In fact, in the first condition, the driver is now projected towards the manoeuvre and, therefore, accepts even smaller gaps. In the case of the second type of passing manoeuvre, however, the driver, stopping behind the heavy vehicle, must consider that the time necessary to carry out the manoeuvre corresponds to the sum of the latter and that necessary for its restart. Consequently, he/she is forced to carry out the manoeuvre only where there are larger gaps. In addition, for the first type of manoeuvre, it should be considered that being carried out entirely in the dynamic phase, the driver could also have greater difficulty in assessing the available gaps.

A two-way ANOVA was conducted to examine the effects of type of passing manoeuvre and LOS on the gap and TTC values. For the analysis of both dependent variables, outliers were assessed by inspection of a boxplot, normality 

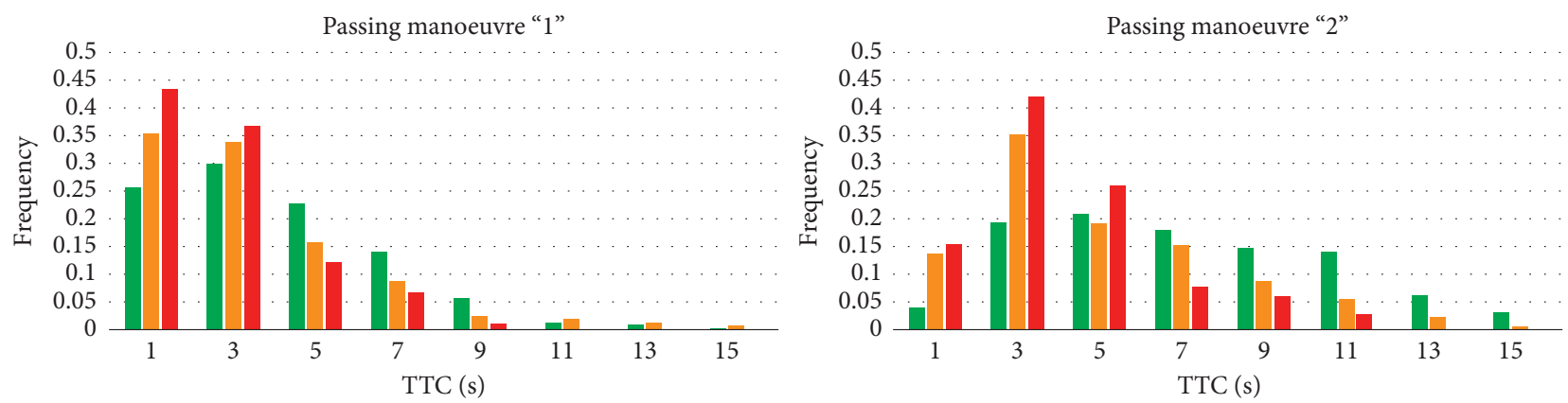

- LOS A

- LOS A

$\because \operatorname{LOS} B$

$\because \operatorname{LOS} \mathrm{B}$

- LOS C

- LOS C

Figure 6: Histograms of TTC for recorded passing manoeuvre of types "1" and "2."
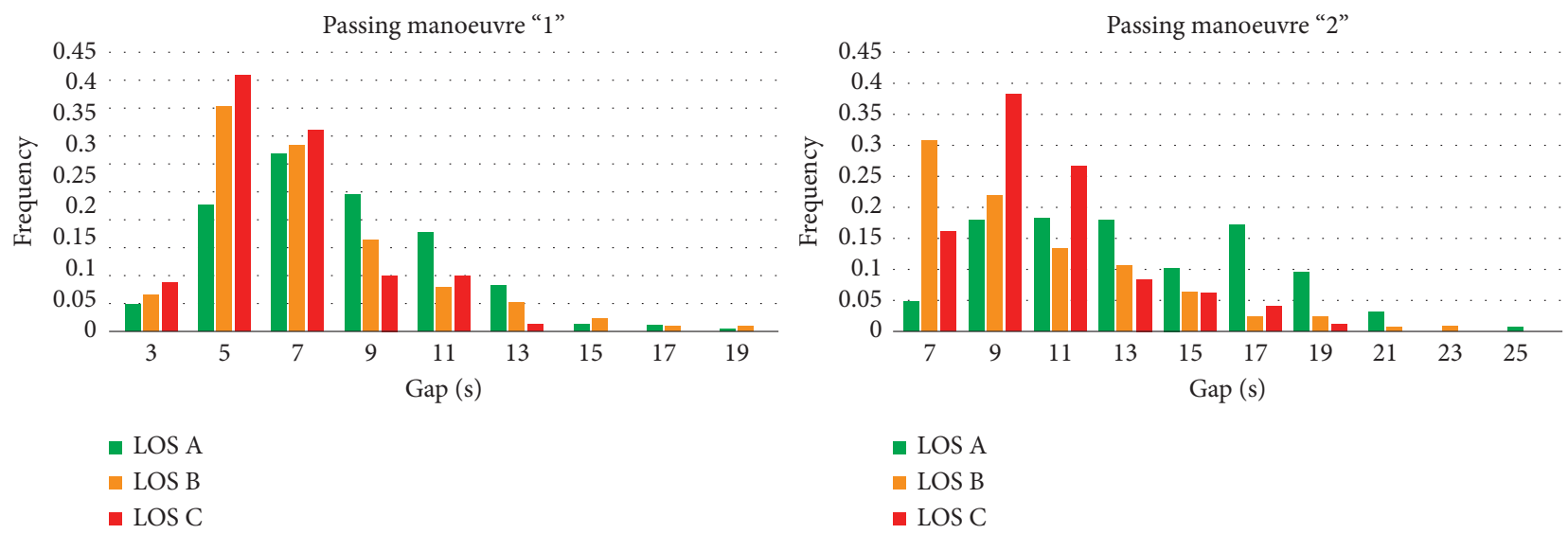

FIgURE 7: Histograms of gap distributions for recorded passing manoeuvre of types "1" and " 2 ."

TABLE 7: Mean value and standard deviation of the gap, TTC, and risky manoeuvres.

\begin{tabular}{lcccccrrr}
\hline \multirow{2}{*}{ Type of passing manoeuvre } & \multirow{2}{*}{ LOS } & \multicolumn{2}{c}{ Gap } & \multicolumn{2}{c}{ TTC } & \multicolumn{3}{c}{ TTC < s } \\
& & MV (s) & SD (s) & MV (s) & SD (s) & N (\%) & MV (s) & SD (s) \\
\hline \multirow{2}{*}{1} & A & 8.12 & 2.79 & 4.08 & 2.68 & $95(25.5)$ & 1.10 & 0.58 \\
& B & 7.21 & 2.84 & 3.37 & 2.67 & $56(35.2)$ & 1.09 & 0.59 \\
& C & 6.47 & 2.02 & 2.64 & 1.92 & $39(43.3)$ & 1.07 & 0.53 \\
\hline & A & 13.63 & 3.98 & 6.98 & 3.47 & $5(3.9)$ & 1.56 & 0.25 \\
& B & 11.36 & 3.48 & 4.90 & 3.05 & $25(13.6)$ & 1.30 & 0.36 \\
& C & 10.42 & 2.65 & 4.02 & 2.34 & $28(15.5)$ & 1.19 & 0.50 \\
\hline
\end{tabular}

$1=$ flying pass manoeuvre; 2 = overtaking manoeuvre, observed after queuing behind the stationary vehicle.

was assessed using Shapiro-Wilk's normality test for each cell of the design, and homogeneity of variances was assessed by Levene's test. There were no outliers, residuals were normally distributed $(P>0.05)$, and there was homogeneity of variances $(P>0.05)$. The findings of the statistical test revealed that the interaction effect between the type of passing manoeuvre and LOS on both the gap and TTC values was statistically significant, respectively $(F(2,1120)=$ $6.787, P=0.001$; partial $\left.\eta^{2}=0.012\right)$ and $(F(2,1110)=6.787$, $P \leq 0.001$; partial $\left.\eta^{2}=0.014\right)$. Therefore, an analysis of simple main effects for type of passing manoeuvre and LOS was performed. There was a statistical significance in mean "gap" values for both factors $(F(1,1120)=529.957, P<0.0001$; partial $\left.\eta^{2}=0.321\right)$ and $(F(2,1120)=53.631, P<0.0001$; partial $\left.\eta^{2}=0.087\right)$ and there was a statistical significance in mean "TTC" values for both factor $(F(1,1110)=115.495$, $P<0.0001$; partial $\left.\eta^{2}=0.094\right)$ and $(F(2,1110)=51.616$, $P<0.0001$; partial $\eta^{2}=0.085$ ). For both dependent variables (gap and TTC) and factors, all pairwise comparisons were run, where the reported 95\% confidence interval and $P$ value are Bonferroni-adjusted. These analyses showed that, for the type of manoeuvre factor, a statistical difference was recorded for each LOS considered (Table 8).

Regarding the LOS factor, the results showed that there was a statistical difference within the type "2" manoeuvre between all LOS groups, while this was not recorded between 
TABLE 8: Results of the pairwise comparison test ( $P$ value) for the type of passing manoeuvre factor.

\begin{tabular}{|c|c|c|c|c|c|}
\hline \multicolumn{2}{|c|}{ Dependent variable } & \multicolumn{2}{|c|}{ Gap } & \multicolumn{2}{|c|}{ TTC } \\
\hline LOS & Type of passing manoeuvre & "1" & “2” & "1" & “2” \\
\hline \multirow{2}{*}{ LOS A } & "1" & - & $<0.0001$ & - & $<0.0001$ \\
\hline & “2” & $<0.0001$ & - & $<0.0001$ & - \\
\hline \multirow{2}{*}{ LOS B } & “1” & - & $<0.0001$ & - & $<0.0001$ \\
\hline & “2” & $<0.0001$ & - & $<0.0001$ & - \\
\hline \multirow{2}{*}{ LOS C } & "1" & - & $<0.0001$ & - & $<0.0001$ \\
\hline & “2” & $<0.0001$ & - & $<0.0001$ & - \\
\hline
\end{tabular}

Boldface indicates statistically significant values with a $5 \%$ level of significance.

LOS B and LOS C as regards the type " 1 " manoeuvre (Table 9). These results confirm that, on two-lane two-way rural roads, the passing manoeuvre is strongly affected by the gap between two successive vehicles on the opposing lane. Therefore, the probability of a safe manoeuvre, especially a flying pass manoeuvre, strongly depends on the LOS that the road infrastructure can offer.

5.2.3. Comparative Analysis. Before presenting the results of the comparative analysis, the main results, obtained using exclusively all the default values of the Aimsun, are reported below. The data set, from this traffic microsimulations, included a total of 831 passing gap observations. In 232 (28\%) of these gaps, the passing manoeuvre type " 1 " was performed, while in the remaining 599 cases (72\%), a type " 2 " overtaking manoeuvre was observed. In this case, a different trend was recorded from that observed in step 2, where some input values were adjusted with the data recorded during the experiments in virtual reality: the number of fly manoeuvres is lower than those carried out following a stop behind the stationary vehicle.

Table 10 shows summary statistics of passing manoeuvres observed on the basis of the three LOS considered. Overall, also, in this case, the results showed that, as traffic flow increases, the percentage of type " 1 " passing manoeuvres decreases. In this situation, however, it is observed that even in the presence of a free-flow traffic condition (LOS A), in which users are practically unaffected by the presence of other vehicles on the road section, the percentage of vehicles that performed a type " 2 " passing manoeuvre is higher than those that performed a type " 1 " passing manoeuvre.

The Chi-squared test results showed that there was a statistically significant association between traffic conditions and the type of passing manoeuvre performed (flying pass/ pass after stopping behind the stopped vehicle) $\left(\chi^{2}(2)=\right.$ 94.673 and $P<0.0001)$, even when using only default software input data. The association was quite strong, according to Cohen [50] (Cramer's $\mathrm{V}=0.338$ ). Therefore, it can be said that the traffic-flow conditions significantly affect the passing manoeuvre approaching the heavy stopped vehicle.

Developing the same analysis performed in step 2, the results (in terms of gap and TTC values, as well as these relating to manoeuvres considered at risk) summarized in
Table 11 were obtained. In this case, also, it was found that, as traffic increases, the TTC values are considerably reduced, and the percentage of manoeuvres considered at risk increases. Moreover, as recorded during step 2 the results showed that, for passing manoeuvre "1," greater manoeuvres at risk were recorded compared to those recorded in passing manoeuvre " 2 ," in all traffic-flow conditions.

Unlike what was obtained from the simulations carried out in step 2, a difference was recorded between the types " 1 " and " 2 " of manoeuvre in terms of manoeuvres considered at risk; for the first type, there is a greater increase in these manoeuvres from LOS A to LOS C compared to the second type of manoeuvre. Specifically, considering the type " 1 " manoeuvre, there was an increase of approximately $10 \%$ between the LOS A configuration and that of LOS B and between the LOS B configuration and LOS $\mathrm{C}$ of approximately $7 \%$. While for the second type of manoeuvre, the increases recorded between the different LOS conditions considered are always negligible (about $2 \%$ between LOS A and LOS B and 1\% between LOS B and LOS C). However, the value of the percentage of manoeuvres considered risky recorded using only the default data of the Aimsun was lower than that obtained using data adjusted according to what was recorded in the virtual reality experiments.

All this highlights that the results obtained with the software default input values differ, in a more precautionary way, compared to those recorded in which some input parameters were adjusted according to the driving behaviour recorded during experiments conducted with a driving simulator. Specifically, it appears that the use of the Aimsun software with its default input values produces a more precautionary situation in terms of safety (in this study quantified in terms of reducing the number of overtaking manoeuvres considered at risk), but more critical in terms of functionality, or the level of service offered (increase in type " 2 " overtaking and therefore increase in waiting times, queuing times, etc.). This could be a consequence of the fact that these tools are created primarily to simulate road traffic in detail (both in the city and on the motorway) to develop more efficient mobility management strategies, rather than to perform road safety analyses. Therefore, although they can potentially be very powerful tools for also performing road safety analyses, in these cases, it seems appropriate that since the variability of driving behaviour plays a determining role, some input parameters are set after their careful evaluation to provide reliable results. 
TABLE 9: Results of the pairwise comparison test ( $P$ value) for the LOS factor.

\begin{tabular}{|c|c|c|c|c|c|c|c|}
\hline \multicolumn{2}{|c|}{ Dependent variable } & \multicolumn{3}{|c|}{ Gap } & \multicolumn{3}{|c|}{ TTC } \\
\hline Type of passing manoeuvre & LOS & LOS A & LOS B & LOS C & LOS A & LOS B & LOS C \\
\hline \multirow{3}{*}{ "1" } & LOS A & - & 0.004 & $<0.0001$ & - & 0.020 & $<0.0001$ \\
\hline & LOS B & 0.004 & - & 0.190 & 0.020 & - & 0.132 \\
\hline & LOS C & $<0.0001$ & 0.190 & - & $<0.0001$ & 0.132 & - \\
\hline \multirow{3}{*}{ " $2 "$} & LOS A & - & $<0.0001$ & $<0.0001$ & - & $<0.0001$ & $<0.0001$ \\
\hline & LOS B & $<0.0001$ & - & 0.009 & $<0.0001$ & - & 0.007 \\
\hline & LOS C & $<0.0001$ & 0.009 & - & $<0.0001$ & 0.007 & - \\
\hline
\end{tabular}

Boldface indicates statistically significant values with a $5 \%$ level of significance.

TABLE 10: Type of manoeuvres performed according to the different LOS analysed.

\begin{tabular}{|c|c|c|c|c|c|c|}
\hline \multirow{3}{*}{ LOS } & \multicolumn{4}{|c|}{ Type of passing manoeuvre } & \multirow{2}{*}{\multicolumn{2}{|c|}{ Total passing manoeuvre }} \\
\hline & \multicolumn{2}{|c|}{1} & \multicolumn{2}{|c|}{2} & & \\
\hline & $N$ & $\%$ & $N$ & $\%$ & $N$ & $\%$ \\
\hline A & 159 & 45.3 & 192 & 54.7 & 351 & 100 \\
\hline B & 51 & 18.5 & 225 & 81.5 & 276 & 100 \\
\hline $\mathrm{C}$ & 22 & 10.8 & 182 & 89.2 & 204 & 100 \\
\hline
\end{tabular}

1 = flying pass manoeuvre; 2 = overtaking manoeuvre, observed after waiting behind the stationary vehicle.

TABLE 11: Mean value and standard deviation of the gap, TTC, and risky manoeuvres.

\begin{tabular}{lcccccccc}
\hline \multirow{2}{*}{ Type of passing manoeuvre } & \multirow{2}{*}{ LOS } & \multicolumn{2}{c}{ Gap } & \multicolumn{3}{c}{ TTC } & \multicolumn{3}{c}{ TTC <2s } \\
& & MV (s) & SD (s) & MV (s) & SD (s) & N (\%) & MV (s) & SD (s) \\
\hline \multirow{3}{*}{1} & A & 10.16 & 2.52 & 5.89 & 2.50 & $9(5.7)$ & 1.44 & 0.34 \\
& B & 9.25 & 2.94 & 5.29 & 2.88 & $8(15.7)$ & 1.48 & 0.41 \\
& C & 7.72 & 2.65 & 4.03 & 2.59 & $5(22.73)$ & 1.68 & 0.24 \\
\hline & A & 14.54 & 3.15 & 8.99 & 2.64 & $0(0.0)$ & - & - \\
& B & 13.30 & 3.46 & 7.93 & 2.90 & $4(1.8)$ & 1.50 & 0.19 \\
& C & 12.76 & 3.32 & 7.42 & 2.79 & $5(2.8)$ & 1.25 & 0.39 \\
\hline
\end{tabular}

1 = flying pass manoeuvre; 2 = overtaking manoeuvre, observed after waiting behind the stationary vehicle.

TABLE 12: Result of statistical test for the GAP and TTC values recorded in the two microscopic traffic simulations performed.

\begin{tabular}{|c|c|c|c|c|c|c|}
\hline \multirow{2}{*}{ GAP } & \multicolumn{3}{|c|}{ Passing manoeuvre type "1" } & \multicolumn{3}{|c|}{ Passing manoeuvre type "2" } \\
\hline & $|t|$ & $P$ value & Result of test & $|t|$ & $P$ value & Result of test \\
\hline LOS A & 7.975 & $<0.001$ & H0 reject & 2.272 & 0.024 & H0 reject \\
\hline LOS B & 4.429 & $<0.001$ & H0 reject & 5.633 & $<0.001$ & H0 reject \\
\hline LOS C & 2.445 & 0.016 & H0 reject & 7.418 & $<0.001$ & H0 reject \\
\hline \multirow{2}{*}{ TTC } & \multicolumn{3}{|c|}{ Passing manoeuvre type “1” } & \multicolumn{3}{|c|}{ Passing manoeuvre type "2" } \\
\hline & $|t|$ & $P$ value & Result of test & $|t|$ & $P$ value & Result of test \\
\hline LOS A & 7.290 & $<0.001$ & H0 reject & 5.887 & $<0.0001$ & H0 reject \\
\hline LOS B & 4.375 & $<0.001$ & $\mathrm{H} 0$ reject & 10.251 & $<0.0001$ & $\mathrm{H} 0$ reject \\
\hline LOS C & 2.854 & 0.005 & H0 reject & 12.535 & $<0.001$ & H0 reject \\
\hline
\end{tabular}

The two data sets (the one related to all the default values and the other one obtained with some input values adjusted by driving simulation experiment) have been statistically analysed to determine if these differences were statistically significant. For both dependent variables (gap and TTC) 6 independent-samples $t$-test were run: one for each LOS and type of manoeuvre considered ( 3 LOS $x 2$ types of manoeuvre). There were no outliers in the data, as assessed by inspection of a boxplot. The gap and TTC values for each level of LOS and type of manoeuvre were normally distributed, as assessed by Shapiro-Wilk's test $(P>0.05)$, and variances were homogeneous, as assessed by Levene's test for equality of variances $(P>0.05)$.

The results of statistical tests revealed that, at each level considered, the differences recorded in the two data sets (driving simulator input data and Aimsun default input data) were statistically significant (Table 12).

\section{Conclusions}

This study aimed to examine the influence of different traffic conditions on passing manoeuvres on rural two-lane two- 
way rural roads, focusing on the end of the manoeuvre considered, using a combined simulation approach (driving simulator and traffic microsimulation).

The results showed that the passing manoeuvre is significantly conditioned on the gap between two successive vehicles on the opposing lane and, as presumably expected, the traffic condition significantly affects the type of manoeuvre performed. In fact, as traffic increases, the percentage of performed fly manoeuvres decreases (LOS A $74.3 \%$, LOS B $46.4 \%$, and LOS C $33.2 \%$ ), a trend due to the occurrence of a smaller number of gaps of considerable width. This highlighted that a medium traffic-flow condition (LOS B) is sufficient to make a fly manoeuvre a less frequent event: compared to the condition of LOS A, a decrease of about 38\% was recorded. The results obtained from traffic microsimulation confirm what had already emerged from the qualitative analysis of the data recorded through the driving simulator. It was found that over $50 \%$ of the participants in the experimentation in virtual reality did not take the risk of overtaking in the condition characterized by the minimum gap, which was strictly necessary to physically manoeuvre (corresponding to $8 \mathrm{~s}$ ).

It was also found that at the end of the passing manoeuvre, the recorded behaviour is very different depending on the variations in the traffic conditions. Clearly, as vehicular flow increases, there are fewer gaps of sufficient width to ensure the possibility of performing the manoeuvre safely; therefore, it was recorded that after having waited for a certain time for the useful gap to carry out the manoeuvre, users are willing to accept gaps of a reduced width compared to that deemed appropriate. Consequently, the TTC values are significantly reduced and the percentage of manoeuvres considered at risk $(\mathrm{TTC}<2 \mathrm{~s})$ increases. From a traffic condition of LOS A to one of LOS B, there was an increase of about $10 \%$ for both types of manoeuvre, even if a higher percentage of risky overtaking was recorded, higher in the case when fly manoeuvre was carried out (about 25\% compared to the other type): the user, already oriented to carry out the manoeuvre, tends to accept even smaller gaps. The results obtained confirmed the trend already observed in the data recorded in virtual reality, where even in that case, as the gap decreases, the overtaking considered at risky increased.

These results highlight that it would be advisable to reduce the interference caused by traffic to avoid unsafe behaviour during passing manoeuvres. Therefore, it might be appropriate to review some design requirements for the type of roads examined so that these also consider the performance of the road infrastructure under prevailing traffic conditions and these are not limited only to the use of design standards (such as passing sight distance) which are not correlated to the traffic condition. In this context, it could be useful to consider further design measures/solutions that meet the specific traffic demand of that specific road. For example, it would be appropriate to limit the longitudinal grade, add additional lanes for slow-moving vehicles, review the location on the road layout of emergency lay-bays, especially where the amount of heavy vehicles is significant, and it might lead to not only a significant reduction in the level of service but also the probability that situations such as the one simulated in this study (stationary heavy vehicle obstructing the entire traffic lane) may arise.

Furthermore, the comparative analysis highlighted that there is a statistically significant difference between the results obtained from the traffic simulations, in which some input parameters of the microscopic passing manoeuvre model for two-lane rural roads were adjusted, with the data recorded in the virtual reality experiment and those that were instead collected from traffic microsimulations using all the software's default input data. Specifically, the results obtained from microsimulations (without adjustment) that have been adopted on the input data seem to differ from those, in which this was carried out more critically, in terms of the level of service offered by the road infrastructure (increase in waiting times, queuing times, etc.) and more precautionary in terms of road safety (in terms of reducing the number of overtaking manoeuvres considered at risk). This could be a consequence of the fact that these types of software were born and widely used and consequently validated to perform traffic management assessments, rather than road safety analyses. Therefore, since the variability of driving behaviour plays a decisive role, especially in complex driving environments such as rural roads, it seems appropriate that the input parameters that can be most influenced by the driver behaviour are set following a careful evaluation to the reality we want to represent.

Although the results obtained are promising and have allowed us to reach useful conclusions, this study presents some limitations that deserve further research in several directions and that it is worth mentioning.

The results obtained refer exclusively to the driving environments and situations considered: a road section in straight, flat, and excellent weather conditions. Therefore, further research efforts are necessary to generalize the results obtained by considering a wider range of driving environments and situations, including examining the impact of some geometric characteristics passing behaviour, such as vertical alignment, pavement quality, visual distances, and roadside characteristics.

The sample was one of convenience and representative only of the Tuscan driver population; as such, it may not be representative of the broader population of drivers. Therefore, further investigations will be performed to evaluate the impact of different driver groups (such as young and old drivers, drivers with different personality traits: anxious, aggressive, etc.) on the influence of traffic conditions on passing behaviour.

Finally, in order to develop predictive models which include traffic volumes, it may be appropriate to analyse other situations, such as overtaking of a moving vehicle.

Despite these limitations, the results obtained clearly show the effect of traffic on passing manoeuvre and promise to also guide the development of initiatives to improve the safety on two-lane two-way rural roads. 


\section{Data Availability}

All data supporting the results are archived and kept by the Department of Civil and Environmental Engineering, Road Safety and Accident Reconstruct Laboratory (LaSIS).

\section{Conflicts of Interest}

The authors declare no conflicts of interest.

\section{References}

[1] OECD, Road Safety Annual Report 2019, IRTAD, OECD/ITF, Paris, France, 2019.

[2] National Center for Statistics and Analysis, State Traffic Data: 2017 data, (Traffic Safety Facts. Report No. DOT HS 812 780), National Highway Traffic Safety Administration, Washington, DC, USA, 2019.

[3] Bureau of Infrastructure Transport and Regional Economics (BITRE), Road Trauma Australia 2015 Statistical Summary, Commonwealth of Australia, Canberra, Australia, 2016.

[4] European Commission, Annual Accident Report 2018, European Commission, Brussels, Belgium, 2018, https://ec. europa.eu/transport/road_safety/sites/roadsafety/files/pdf/ statistics/dacota/asr2018.pdf.

[5] ACI-ISTAT, Incidenti Stradali in Italia-Anno 2019, ACIISTAT, Rome, Italy, in Italian Language, 2019.

[6] Highway Capacity Manual (HCM), TRB, National Research Council, Washington, DC, USA, 2010.

[7] R. Gray and D. M. Regan, "Perceptual processes used by drivers during overtaking in a driving simulator," Human Factors: The Journal of the Human Factors and Ergonomics Society, vol. 47, no. 2, pp. 394-417, 2005.

[8] G. Hegeman, A. Tapani, and S. Hoogendoorn, "Overtaking assistant assessment using traffic simulation," Transportation Research Part C: Emerging Technologies, vol. 17, no. 6, pp. 617-630, 2009.

[9] S. Jamson, K. Chorlton, and O. Carsten, "Could intelligent speed adaptation make overtaking unsafe?" Accident Analysis \& Prevention, vol. 48, pp. 29-36, 2012.

[10] T. Richter, S. Ruhl, J. Ortlepp, and E. Bakaba, "Causes, consequences and countermeasures of overtaking accidents on two-lane rural roads," Transportation research Procedia, vol. 25, pp. 1989-2001, 2017.

[11] H. Bar-Gera and D. Shinar, "The tendency of drivers to pass other vehicles," Transportation Research Part F: Traffic Psychology and Behaviour, vol. 8, no. 6, pp. 429-439, 2005.

[12] M. M. Z. Á. Török, "Improving traffic flow characteristics by suppressing shared taxis maneuvers," Periodica Polytechnica Transportation Engineering, vol. 44, pp. 69-74, 2016.

[13] A. T. Kashani, E. Ayaziand, and M. S. Ravasani, "Identifying significant variables influencing overtaking maneuvers on two-lane, two-way rural roads in Iran," Periodica Polytechnica Transportation Engineering, vol. 44, no. 3, pp. 155-163, 2016.

[14] A. Polus and A. B. Tomecki, "Passing experiment on two-lane rural highways," Transportation Research Record: Journal of the Transportation Research Board, vol. 1112, pp. 115-123, 1987.

[15] G. Asaithambi and G. Shravani, "Overtaking behaviour of vehicles on undivided roads in non-lane based mixed traffic conditions," Journal of Traffic and Transportation Engineering (English Edition), vol. 4, no. 3, pp. 252-261, 2017.

[16] A. Polus, M. Livneh, and B. Frischer, "Evaluation of the passing process on two-lane rural highways," Transportation
Research Record: Journal of the Transportation Research Board, vol. 1701, no. 1, pp. 53-60, 2000.

[17] D. W. Harwood and D. K. G. K. R. Richard, "Criteria for passing sight distance for roadway design and marking," Transportation Research Record: Journal of the Transportation Research Board, vol. 2195, pp. 36-46, 2010.

[18] C. Llorca and A. García, "Evaluation of passing process on two-lane rural highways in Spain with new methodology based on video data," Transportation Research Record: Journal of the Transportation Research Board, vol. 2262, no. 1, pp. 42-51, 2011.

[19] A. Shariat-Mohaymany, A. T. Kashani, H. Nosrati, and S. Kazemzadehazad, "Development of head-on conflict model for overtaking maneuvers on two-lane rural roads using inductive loop detectors," Journal of Transportation Safety \& Security, vol. 5, no. 4, pp. 273-284, 2013.

[20] G. Hegeman, S. Hoogendoornand, and K. A. Brookhuis, "Observations overtaking manoeuvres on bi-directional roads," in Advanced OR and AI Methods in Transportation, A. Jaszkiewicz, M. Kaczmarek, J. Zak et al., Eds., pp. 505-510, Publishing House of Poznan University of Technology, Poznan, Poland, 2005.

[21] V. Papakostopoulos, D. Nathanael, E. Portouli, and N. Marmaras, "The effects of changes in the traffic scene during overtaking," Accident Analysis \& Prevention, vol. 79, pp. 126-132, 2015.

[22] V. Branzi, L. Domenichini, and F. La Torre, “Drivers' speed behaviour in real and simulated urban roads-a validation study," Transportation Research Part F: Traffic Psychology and Behaviour, vol. 49, pp. 1-17, 2017.

[23] L. Domenichini, F. La Torre, V. Branzi, and A. Nocentini, "Speed behaviour in work zone crossovers. A driving simulator study," Accident Analysis \& Prevention, vol. 98, pp. 10-24, 2017.

[24] M. Klüver, C. Herrigel, C. Heinrich, H.-P. Schöner, and H. Hecht, "The behavioral validity of dual-task driving performance in fixed and moving base driving simulators," Transportation Research Part F: Traffic Psychology and Behaviour, vol. 37, pp. 78-96, 2016.

[25] A. Knapper, M. Christoph, M. Hagenzieker, and K. Brookhuis, "Comparing a driving simulator to the real road regarding distracted driving speed," European Journal of Transport and Infrastructure Research, vol. 15, no. 2, pp. 205-225, 2015.

[26] G. H. Bham, M. C. Leu, M. Vallati, and D. R. Mathur, "Driving simulator validation of driver behavior with limited safe vantage points for data collection in work zones," Journal of Safety Research, vol. 49, no. 6, pp. 53-60, 2014.

[27] F. Bella, "Driving simulator for speed research on two-lane rural roads," Accident Analysis \& Prevention, vol. 40, no. 3, pp. 1078-1087, 2008.

[28] F. Bella, "How traffic conditions affect driver behavior in passing maneuver," Advances in Transportation Studies, pp. 113-126, 2011.

[29] H. Farah, E. Yechiam, S. Bekhor, T. Toledo, and A. Polus, "Association of risk proneness in overtaking maneuvers with impaired decision making," Transportation Research Part F: Traffic Psychology and Behaviour, vol. 11, no. 5, pp. 313-323, 2008.

[30] S. El-Bassiouniand and T. Sayed, "Design requirements for passing sight distance: a risk based approach," in Proceedings of the 89th Annual Meeting Transportation Research Record, Washington, DC, USA, 2010. 
[31] H. Farah and T. Toledo, "Passing behavior on two-lane highways," Transportation Research Part F: Traffic Psychology and Behaviour, vol. 13, no. 6, pp. 355-364, 2010.

[32] H. Farah, "Modeling drivers' passing duration and distance in a virtual environment," IATSS Research, vol. 37, no. 1, pp. 61-67, 2013.

[33] H. Farahand and C. L. Azevedo, "Safety analysis of passing maneuvers using extreme value theory," IATSS Research, vol. 41, no. 1, pp. 12-21, 2016.

[34] S. M. S. Mahmud, L. Ferreira, M. S. Hoque, A. Tavassoli, and A. Tavassoli, "Micro-simulation modelling for traffic safety: a review and potential application to heterogeneous traffic environment," IATSS Research, vol. 43, no. 1, pp. 27-36, 2019.

[35] J. Archer, Indicators for Traffic Safety Assessment and Prediction and Their Application in Micro-simulation Modelling: A Study of Urban and Suburban Intersections, Royal Institute of Technology, Stockholm, Sweden, 2005.

[36] FHWA, Surrogate Measures of Safety from Traffic Simulation Models, Federal Highway Administration, Washington, DC, USA, 2003.

[37] D. Gettman, L. Pu, T. Sayed, and S. G. Shelby, "Surrogate safety assessment model and validation: final report," TurnerFairbank Highway Research Center, McLean, VA, USA, FHWA-HRT-08-051, 2008.

[38] J. J. L. Rilett, "Application of distributed traffic simulation for passing behavior study," Transportation Research Record: Journal of the Transportation Research Board, vol. 1899, pp. 11-18, 2005.

[39] W. Young, A. Sobhani, M. G. Lenné, and M. Sarvi, "Simulation of safety: a review of the state of the art in road safety simulation modelling," Accident Analysis \& Prevention, vol. 66, pp. 89-103, 2014.

[40] H. M. Al-Ahmadi, A. Jamal, I. Reza, K. Assi, and S. A. Ahmed, "Using microscopic simulation-based analysis to model driving behavior: a case study of Khobar-Dammam in Saudi Arabia," Sustainability, vol. 11, no. 3018, 2019.

[41] H. Farah, S. Bekhor, and A. Polus, "Risk evaluation by modeling of passing behaviour on two-lane rural highways," Accident Analysis and Prevention, vol. 41, no. 4, pp. 87-89, 2009.

[42] TSS, Aimsun Version 8.0.2 User Manual, Transport Simulation Systems, Barcelona, Spain, 2013.

[43] C. Llorca, A. T. Moreno, A. Lenorzer, J. Casas, and A. García, "Development of a new microscopic passing maneuver model for two-lane rural roads," Transportation Research Part C: Emerging Technologies, vol. 52, pp. 157-172, 2015.

[44] L. Domenichini, F. La Torre, D. Vangi, A. Virga, and V. Branzi, "Influence of the lighting system on the driver's behavior in road tunnels. A driving simulator study," Journal of Transportation Safety and Security, vol. 9, no. 2, pp. 16-23, 2017.

[45] L. Domenichini, V. Branzi, and M. Meocci, "Virtual testing of speed reduction schemes on urban collector roads," Accident Analysis \& Prevention, vol. 110, pp. 38-51, 2018.

[46] L. Domenichini, V. Branzi, and M. Smorti, "Influence of drivers' psychological risk profiles on the effectiveness of traffic calming measures," Accident Analysis \& Prevention, vol. 123, pp. 243-255, 2019.

[47] Ministry of Infrastructures and Transports, "Nuovo Codice Della Strada," D.L. 30 Aprile 1992 n.285 e Successive Modificazioni. Gazzetta Ufficiale della Repubblica Italiana, n. 114. MIT, Via G. Caraci 36, Roma, 1992, in Italian Language.

[48] Ministry of Infrastructures and Transports, "Norme Funzionali e Geometriche Per La Costruzione Delle Strade,"
Decreto Ministeriale n.6792 del 05/11/2001. MIT, Via G. Caraci 36, Roma, 2001, in Italian Language.

[49] D. Vangi, Ricostruzione Della Dinamica Degli Incidenti Stradali-Principi e Applicazioni, Firenze University Press, Nelson Mandela Bay, South Africa, 2008.

[50] J. Cohen, Statistical Power Analysis for the Behavioural Sciences, PsychologyPress, New York, NY, USA, 2nd edition, 1988.

[51] M. Losa, F. Frendo, A. Cofrancesco, and R. Bartolozzi, "Procedure for validating fixed base driving simulators," Transport, vol. 28, no. 2, pp. 420-430, 2013.

[52] AASHTO, Highway Safety Manual, American Association of State and Highway Transportation Officials, Washington, DC, USA, 1st edition, 2010.

[53] K. Basak, S. N. Hetu, Z. Li et al., "Modeling reaction time within a traffic simulation model," in Proceedings of the 16th International IEEE Conference on Intelligent Transportation Systems (ITSC 2013), pp. 302-309, Hague, Netherlands, 2013.

[54] P. S. Bokare and A. K. Maurya, "Acceleration-deceleration behaviour of various vehicle types," Transportation Research Procedia, vol. 25, pp. 4733-4749, 2017.

[55] A. Svensson, AMethod for Analysing the Traffic Process in a Safety Perspective, Lund University, Lund, Sweden, 1998.

[56] Å. Svensson and C. Hydén, "Estimating the severity of safety related behaviour," Accident Analysis \& Prevention, vol. 38, no. 2, pp. 379-385, 2006.

[57] A. Dijkstra and J. Drolenga, "Safety effects of route choice in a road network: simulation of changing route choice," Research in the Framework of the European Research Programme InSafety, SWOV Institute for Road Safety Research, Hague, The Netherlands, 2008.

[58] R. J. Kiefer, C. A. Flannagan, C. Jerome, and C. J. Jerome, "Time-to-collision judgments under realistic driving conditions," Human Factors: The Journal of the Human Factors and Ergonomics Society, vol. 48, no. 2, pp. 334-345, 2006.

[59] A. Shariat-Mohaymany, A. Tavakoli-Kashani, H. Nosrati, and A. Ranjbari, "Identifying significant predictors of head-on conflicts on two-lane rural roads using inductive loop detectors data," Traffic Injury Prevention, vol. 12, no. 6, pp. 636-641, 2011.

[60] G. Hegeman, Assisted Overtaking: An Assessment of Overtaking on Two-Lane Rural Roads, Netherlands Research School for Transport, Delft, The Netherlands, 2008.

[61] Y. Hassan, S. M. Easa, A. O. Abd El Halim, and A. O. A. El Halim, "Passing sight distance on two-lane highways: review and revision," Transportation Research Part A: Policy and Practice, vol. 30, no. 6, pp. 453-467, 1996.

[62] J. Glennon, "New and improved model of passing sight distance," Transportation Research Record: Journal of the Transportation Research Board, vol. 1195, pp. 55-69, 1988. 